NOTICE: THIS MATERIAL MAY BE

PROTECTED BY COPYRIGHT LAW

(TITLE 17 U.S. COOE)

Underwater Optical Measurement of Position and

Orientation Using a Prepared, Passive Target

by

Niel Raymond Nickolaisen
B.S., Physics, Utah State University (1983)
Submitted in partial fulfillment of the requirements for the degree of
MASTER OF SCIENCE IN OCEAN ENGINEERING at the
MASSACHUSETTS INSTITUTE OE TECHNOLOGY and the
WOODS HOLE OCEANOGRAPHIC INSTITUTION

September, 1989

\begin{tabular}{c} 
MANINE \\
BIOLOGICAL \\
LABORATORY \\
\hline LIBRARY \\
\hline $\begin{array}{c}\text { WOOOS HOLE, MASS. } \\
\text { W. H. O. L. }\end{array}$ \\
\hline
\end{tabular}

Copyright Niel Raymond Nickolaisen, 1989 All rights reserved.

The author hereby grants to MIT and WHOI permission to

reproduce and to distribute copies of this thesis document in whle of in part., ,

Signature of Author ......

Joint rrogram in Dceanographic Engineering

Massachusetts Institute of Technology Woods Hole Cceanographic Institution 1 .

September 15, 1989

Certified by

Dr. Dana R. Yoerger Woods Hole oceanographic Institution

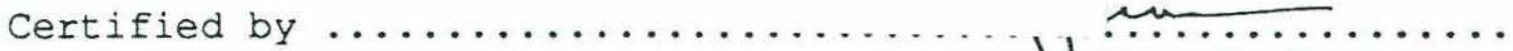
Dr. Althur B. Baggeroer Massachusetts Institute, of Technology Accepted by

Chairman, Joint Committee for Oceanographic Engineering Massachusetts Institute of Technology/ woods Hole Oceanographic Institution

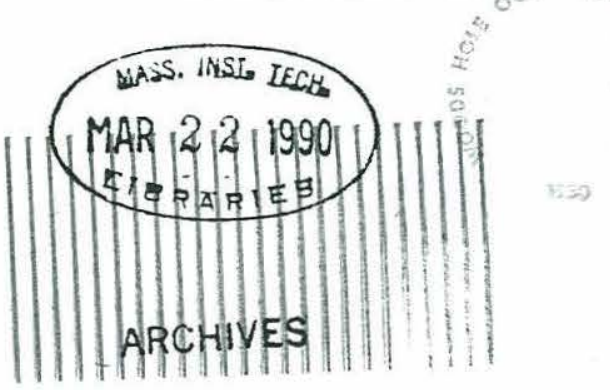




\title{
Underwater Optical Measurement of Position and orientation Using a Prepared, Passive Target
}

\author{
by
}

Niel Raymond Nickolaisen

Submitted to the Massachusetts Institute of Technology/hoods Hole Oceanographic Institution Joint Program in Oceanographic Engineering on September 15, 1989 in partial fulfillment of the requirements for the degree of Master of Science in Dcean Engineering.

\begin{abstract}
In order to provide an accurate and efficient method of docking, station keeping, and navigation for the JASON remotely operated vehicle, an optical tracking method is investigated. The method needs to be accurate enough for underwater control and fast enough to enable timely control. The method presented solves the camera location problem in a closed form and is accurate for accurate measurements of image plane coordinates. Target design criteria are discussed and a prepared, passive target is selected. Testing and error analysis reveal that the approach gives good results for camera lenses with focal lengths greater than $4.8 \mathrm{~mm}$. The effects of underwater use are discussed and a non-numerical method of compensating for the underwater effects is presented. The compensation method shifts the image plane coordinates toward the image plane center and gives results that are within acceptable error margins.

Thesis Supervisor: Dr. Dana R. Yoerger

Woods Hole oceanographic Institution
\end{abstract}




\section{Acknowledgements}

I am very appreciative to Dr. Dana Yoerger for his help and patience. He provided the right insights at the right times. I would also like to thank Rikki Razdan and Alan M. Kielar at ISCAN and I hope that others may see the potential their tracking systems have in underwater applications. I thank the United States Navy, the Massachusetts Institute of Technology, and the woods Hole oceanographic Institution for the opportunity to participate in the Joint Program. Most of all, I thank my wife, Karen, for her encouragement and support. 
Ta le of Contents

\begin{tabular}{|c|c|c|}
\hline Chapter & 1 & Introduction \\
\hline Chapter & 2 & Use of Perspective Transformations \\
\hline & 2.2 & $\begin{array}{l}\text { Detailed Analysis of the Use of } \\
\text { Perspective Transformations to } \\
\text { Determine RoV Position } \\
\text { Determining the Transformation } \\
\text { Matrix }\end{array}$ \\
\hline Chapter & 3 & Use of Prepared, Passive Targets \\
\hline & $\begin{array}{l}3.1 \\
3.2\end{array}$ & $\begin{array}{l}\text { Target Design Criteria } \\
\text { Target Spot Placement }\end{array}$ \\
\hline Chapter & 4 & $\begin{array}{l}\text { Description of the ISCAN Video } \\
\text { Tracking System }\end{array}$ \\
\hline Chapter & 5 & Testing \\
\hline & $\begin{array}{l}5.1 \\
5.2 \\
5.3\end{array}$ & $\begin{array}{l}\text { Test Design } \\
\text { Camera Calibration } \\
\text { Test Results }\end{array}$ \\
\hline Chapter & 6 & Underwater Effects \\
\hline Chapter & 7 & Conclusions \\
\hline Figures & & \\
\hline Appendic & & \\
\hline Bibli & & \\
\hline
\end{tabular}




\section{Lis: of Figures}

\begin{tabular}{|c|c|c|}
\hline Eigure & 1 & ARGO/JASON Navigation Referenced to Ship \\
\hline Eigure & 2 & JASON Navigation Referenced to ARGO \\
\hline igure & 3 & JASON Navigation Referenced to Seafloor \\
\hline igure & 4 & Displacement of Camera Coordinate System \\
\hline igure & 5 & Pan Angle Rotation \\
\hline igure & 6 & Tilt Angle Rotation \\
\hline igure & 7 & Swing Angle Rotation \\
\hline Eigure & 8 & Possible Target Spot Location \\
\hline igure & 9 & Test Target Base Plate \\
\hline igure & 10 & Error Versus Range (8mm lens in air) \\
\hline igure & 11 & $\begin{array}{c}\text { Error Versus Range (uncorrected } 8 \mathrm{~mm} \text { lens } \\
\text { in water) }\end{array}$ \\
\hline Eigure & 12 & $\begin{array}{c}\text { Error Versus Range (corrected } 8 \mathrm{~mm} \text { lens } \\
\text { in water) }\end{array}$ \\
\hline Eigure & 13 & Error Versus Range ( $12 \mathrm{~mm}$ lens in air) \\
\hline Eigure & 14 & $\begin{array}{c}\text { Error Versus Range (uncorrected } 12 \mathrm{~mm} \text { lens } \\
\text { in water) }\end{array}$ \\
\hline igure & 15 & $\begin{array}{c}\text { Error Versus Range (corrected } 12 \mathrm{~mm} \text { lens } \\
\text { in water) }\end{array}$ \\
\hline
\end{tabular}




\section{Chapter 1 Introduction}

This paper and the associated research involve work done on a visual tracking and control system for the JASON remotely operated vehicle (ROV). JASON is part of the JASON/ARGO project. The problem, stated simply, is the following: Is it possible to visually track JASON and use information about its position to control JASON in station keeping, docking, and navigation procedures?

The key to accomplishing this task is being able to accurately determine the position of the ROV in its underwater environment. I attempt to find JASON's position by using a method of perspective transformations and by using a spot tracker invented by engineers at ISCAN Corp in Cambridge, MA.

The ARGO/JASON system is an advanced scientific tool that is designed to explore the ocean floor. The ARGO sled carries advanced sonar and video systems that scan the ocean floor. When the ARGO "discovers" something of interest and the systein operators want to take a closer look at the "discovery", the JASON ROV is dispatched from a port in ARGO. Aboard ship, the operator maneuvers JASON manually or automatically using various acoustic navigation references.

Currently, JASON's position can be determined by several 
different acoustic references. These are summarized in figures 1,2 , and 3 .

The first is a low frequency long baseline system that is also used to navigate ARGO and the surface ship. While the system works over a long range (up to $10,000 \mathrm{~m}$ ), it suffers from modest precision (plus or minus $10 \mathrm{~m}$ ) and a low update rate (one fix every ten seconds). Also, JASON can easily be out of sight of the transponders when operating in rough terrain.

The second navigation reference is a high frequency system called SHARPS. Like the low frequency system, it uses the travel time of acoustic pulses to determine position. Due to the high bandwidth of SHARPS, range precision is about 1 $\mathrm{cm}$. The high frequency of SHARPS $(300 \mathrm{kHz})$ permits update rates of up to 10 fixes per second. A short baseline on JASON is used to position JASON relative to ARGO. SHARPS transponders, currently in development, will be used to navigate JASON relative to a fixed net on the seafloor.

It would simplify the use and control of JASON if it were possible to use $\mathrm{JASON}^{\prime} \mathrm{S}$ on board cameras to determine JASON's position relative to some fixed target or landmark. This position information (and information about the time rate of change of position) could then be used to automatically 
control JASON's thrusters to manuver it into or keep it in a desired position. This would enable the operator to use the manipulator arm without having to also maintain JASON's position against any currents or disturbances. Knowing JASON's position relative to some target or landmark would provide a method of passive navigation. Locating JASON visually relative to an object would be a complementary alternative to the acoustic systems. While inappropriate for mapping and survey, visual navigation could allow JASON to hover close to objects without installing a seafloor net. This capability could be used to aid in manipulation or close-up inspection.

Developing a visual method of controlling JASON depends on whether it is possible to accurately and quickly determine JASON's position. Stated differently, if we know the shape or characteristics of a landmark or target and how that landmark or target looks to JASON's cameras, can we accurately determine how far away and at what angles JASON is from the landmark or target? Furthermore, can we obtain this information quickly enough to control JASON?

These questions were investigated in several steps. Eirst, study the methods of finding a camera's position relative to a target and choose the method that can 
solve the problem quickly and accurately enough to meet the control needs of JASON. Second, based on the method chosen above, design a target or teach the system a landmark that can be used as a reference. Third, test and implement the method and system.

Based on a review of the literature [Duda, 1973], [Ganapathy, 1984], [Haralick, 1980], it appears that perspective transformations provide the best mathematical approach to determining ROV position. The method described here looks at the relationship between target spot coordinates and those spots as they appear on the camera image plane and then uses this relationship to get a closed form solution to the problem. This solution method leads to the selection of a target composed of four spots on a plane. 


\section{Chapter 2}

\subsection{Detailed Analysis of the Use of Perspective Transformations to Determine ROV Position.}

To visually control the ROV, one must know its position relative to the target. This is found by decomposing the problem into a series of coordinate transformations and their associated transformation matrices. The transformations relate the position and orientation of the camera (and ROV) to some fixed point (the target). These transformations move the coordinate axes from the target to the camera center and rotate the axes to line up along the camera's iine of sight.

Two coordinate systems will be used, one for the target and one for the camera (ROV). Let the target coordinate system be labeled $X, Y, Z$. Let the camera be centered at a point $S$ with a coordinate system labeled $X^{\prime}, Y^{\prime}, Z^{\prime}$. Let the point $P$ be where the line of signt of the camera intercepts the $X, Y$ plane. Let the point $O$ be where the line of sight of the camera intercepts the image plane (the $\mathrm{U}, \mathrm{V}$ plane). Point $\mathrm{O}$ is the principal point. Let the line $\mathrm{S}^{\prime} \mathrm{P}$ represent the projection of SP onto the $X, Y$ plane. These coordinate systems, points, and lines are shown in figure 4.

The first step of the coordinate transformation is performed by moving the camera coordinate axes to $\mathrm{S}$. Next, 
rotate the $X^{\prime}, Y^{\prime}, Z^{\prime}$ system around the $Z^{\prime}$ axis by an angle (1) (the pan angle) such that the $Y^{\prime}$ axis is parallel to $S^{\prime} P$ (see figure 5). Now, rotate the $X^{\prime}, Y^{\prime}, Z^{\prime}$ system around the $X^{\prime}$ axis by an angle (m) (the tilt angle) such that the $Z^{\prime}$ axis lines up with SP (see figure 6). As a final step, rotate the $X^{\prime}, Y^{\prime}, Z^{\prime}$ system around the $Z^{\prime}$ axis by an angle ( $n$ ) (the swing angle) such that the $X^{\prime}$ axis is parallel to the $U$ axis of the image plane (see figure 4). After these transformations have been done, the $\mathrm{X}^{\prime}$ axis will be parallel to the $\mathrm{U}$ axis of the image plane, the $Y^{\prime}$ axis will be either parallel or antiparaliel to the $V$ axis of the image plane, and the $Z^{\prime}$ axis will be aligned with SP. Separately, there four steps can be summarized as follows:

1. Moving the origin to s:

$$
\text { [D] = Displacement Matrix }=\left[\begin{array}{cccc}
1 & 0 & 0 & -X_{s} \\
0 & 1 & 0 & -Y_{s} \\
0 & 0 & 1 & -Z_{s} \\
0 & 0 & 0 & 1
\end{array}\right]
$$

Where $\mathrm{X}_{s}, \mathrm{Y}_{s}, \mathrm{Z}_{\mathrm{s}}$ are the coordinates (in the $X, Y, Z$ system) of the point $S$. 
2. Rotating $X^{\prime}, Y^{\prime}, Z^{\prime}$ about $Z^{\prime}$ (pan angle of 1 ):

$$
[R 1]=\text { First Rotation Matrix }=\left[\begin{array}{cccc}
\cos (1) & \sin (1) & 0 & 0 \\
-\sin (1) & \cos (1) & 0 & 0 \\
0 & 0 & 1 & 0 \\
0 & 0 & 0 & 1
\end{array}\right]
$$

3. Rotating $X^{\prime}: Y^{\prime}, Z^{\prime}$ about $X^{\prime}$ (tilt angle of $\mathrm{m}$ ):

$$
\text { [R2] = Second Rotation Matrix }=\left[\begin{array}{cccc}
0 & 0 & 0 & 0 \\
0 & \cos (m) & \sin (m) & 0 \\
0 & -\sin (m) & \cos (m) & 0 \\
0 & 0 & 0 & 1
\end{array}\right]
$$

4. Rotating $X^{\prime}, Y^{\prime}, Z^{\prime}$ about $Z^{\prime}$ (swing angle of $n$ ):

$$
[R 3]=\operatorname{Third} \text { Rotation Matrix }=\left[\begin{array}{cccc}
-\cos (n) & 0 & -\sin (n) & 0 \\
0 & 1 & 0 & 0 \\
\sin (n) & 0 & \cos (n) & 0 \\
0 & 0 & 0 & 1
\end{array}\right]
$$

Combining the rotation steps gives a matrix that relates the three rotation angles: 
$[R]=[R 3][R 2][R 1]$

The elements of [R] can be written as:

$$
[R]=\left[\begin{array}{llll}
a & b & c & 0 \\
d & e & f & 0 \\
g & h & i & 0 \\
0 & 0 & 0 & 1
\end{array}\right]
$$

where: $\quad a=\cos (n) \cos (1)-\sin (1) \sin (m) \sin (n)$

$b=\cos (n) \sin (1)+\cos (1) \sin (m) \sin (n)$

$c=-\cos (m) \sin (n)$

$\mathrm{d}=-\sin (1) \cos (\mathrm{m})$

$e=\cos (1) \cos (m)$

$\mathrm{f}=\sin (\mathrm{m})$

$g=\cos (1) \sin (n)+\sin (1) \sin (m) \cos (n)$

$h=\sin (m) \sin (n)-\cos (1) \sin (m) \cos (n)$

$i=\cos (m) \cos (n)$

The rotation matrix, [R], can be combined with the displacement matrix, [D], to obtain a matrix whose elements contain information about the position of the camera reiative to the target. The resulting matrix will be called [EXT] because it contains information extrinsic to the 
camera.

$[\mathrm{EXT}]=[R][D]$

$[\operatorname{EXT}]=\left[\begin{array}{llll}a & b & c & p \\ d & e & f & q \\ g & h & i & r \\ 0 & 0 & 0 & 1\end{array}\right]$

where $a, b, c, d, e, f, g, h$, and $i$ are the same as before and:

$$
\begin{aligned}
& p=-a X_{s}-b Y_{s}-c Z_{s} \\
& q=-d X_{s}-e Y_{s}-f Z_{s} \\
& r=-g X_{s}-h Y_{s}-i Z_{s}
\end{aligned}
$$

It is worth noting here that homogeneous coordinates are being used which is why there are four rows in the above matrices. Conversion back to the three dimensional coordinate system is done by dividing all other rows by the fourth (this will be covered in detail when discussing the closed form solution to the problem).

Because the measurements of camera position will use images projected onto the image plane, the camera center coordinates $\left(X^{\prime}, Y^{\prime}, Z^{\prime}\right)$ must be converted to coordinates 
centered on the image plane $(U, V)$. Let $u^{\prime}$ and $v^{\prime}$ denote coordinates of a point on the image plane. $u^{\prime}$ and $v^{\prime}$ are related to the $X^{\prime}, Y^{\prime}, Z^{\prime}$ coordinate system by:

$$
\begin{aligned}
& u^{\prime}=\left(x^{\prime} \star F\right) / z^{\prime} \\
& v^{\prime}=\left(y^{\prime} \star E\right) / z^{\prime}
\end{aligned}
$$

where $\mathrm{F}$ is the focal length of the camera and $x^{\prime}, y^{\prime}, z^{\prime}$ are the coordinates of the point on the image plane in the $X^{\prime}, Y^{\prime}, Z^{\prime}$ coordinate system.

Because measurements on the inage plane will be rasterized (in pixels), it is necessary to scale the measurements to get them in terms of units that will be used to measure the sosition of the camera ralative to the target. This can be done by including a factor of $k_{u}$ (in the $U$ direction) and a factor of $k_{\mathrm{v}}$ (in the $\mathrm{V}$ direction). $\mathrm{k}_{\mathrm{u}}$ will be assumed positive but $\mathrm{k}_{\mathrm{v}}$ can be either positive or negative.

Now, with the coordinates of the principal point, 0 , being labeled ( $\left.u_{0}, v_{0}\right)$, the relation between the $X^{\prime}, Y^{\prime}, Z^{\prime}$ coordinate system and the image plane coordinates, $u$ and $v$, can be written as:

$$
\begin{aligned}
& \mathrm{u}=\mathrm{u}_{0}+\left(\mathrm{k}_{\mathrm{u}} \star \mathrm{x}^{\prime} \star \mathrm{F}\right) / \mathrm{z}^{\prime} \\
& \mathrm{v}=\mathrm{v}_{0}+\left(\mathrm{k}_{\mathrm{v}} \star \mathrm{y}^{\prime} \star \mathrm{F}\right) / \mathrm{z}^{\prime}
\end{aligned}
$$


This expression can be simplified by letting $k_{u}{ } F$ be $k_{1}$ and $k_{\mathrm{v}} \star \mathrm{F}$ be $\mathrm{k}_{2}$. Writing this in terms of a homogeneous coordinate system yields the result:

$$
\left[\begin{array}{l}
u \\
v \\
1
\end{array}\right]=\left[\begin{array}{cccc}
k_{1} & 0 & u_{0} & 0 \\
0 & k_{2} & v_{0} & 0 \\
0 & 0 & 1 & 0
\end{array}\right]\left[\begin{array}{l}
x^{\prime} \\
y^{\prime} \\
z^{\prime} \\
1
\end{array}\right]
$$

$$
\text { or, }
$$

$$
\left[\begin{array}{l}
u \\
v \\
1
\end{array}\right]=\left[\begin{array}{ccc}
k_{1} & 0 & u_{0} \\
0 & k_{2} & v_{0} \\
0 & 0 & 1
\end{array}\right]\left[\begin{array}{c}
x^{\prime} \\
y^{\prime} \\
z^{\prime}
\end{array}\right]
$$

The matrix containing $\mathrm{k}_{1}, \mathrm{k}_{2}$, $\mathrm{u}_{0}, \mathrm{v}_{0}$ will be called [INT] since it contains information intrinsic to the camera i.e. focal length, rasterization scaling, and principal point coordinates.

Previously, the $X^{\prime}, Y^{\prime}, Z^{\prime}$ coordinate system was related to the $x, y, z$, system by the [EXT] matrix: 


$$
\left[\begin{array}{l}
x^{\prime} \\
y^{\prime} \\
z^{\prime}
\end{array}\right]=[\mathrm{EXT}]\left[\begin{array}{l}
x \\
y \\
z \\
1
\end{array}\right]
$$

Also, the U,V coordinate system was related to the $\mathrm{X}^{\prime}, \mathrm{Y}^{\prime}, \mathrm{Z}^{\prime}$ system with the [INT] matrix:

$$
\left[\begin{array}{l}
u \\
v \\
1
\end{array}\right]=[\text { INT }]\left[\begin{array}{l}
x^{\prime} \\
y^{\prime} \\
z^{\prime}
\end{array}\right]
$$

These matrices can be combined to relate the $\mathrm{U}, \mathrm{V}$ coordinate system to the $X, Y, Z$ coordinate system (where the target is located):

$$
\left[\begin{array}{l}
\mathrm{u} \\
\mathrm{v} \\
\mathrm{I}
\end{array}\right]=\left[\text { INT] [EXT] }\left[\begin{array}{l}
\mathrm{x} \\
\mathrm{y} \\
\mathrm{z} \\
1
\end{array}\right]\right.
$$




$$
\left[\begin{array}{l}
u \\
v \\
1
\end{array}\right]=[T]\left[\begin{array}{l}
x \\
y \\
z \\
1
\end{array}\right]
$$

where [T] is a $3 \times 4$ matrix whose elements are:

$$
\begin{aligned}
& t_{11}=k_{1} a+u_{0} g \\
& t_{12}=k_{1} b+u_{0} h \\
& t_{13}=k_{1} c+u_{0} i \\
& t_{14}=k_{1} p+u_{0} r \\
& t_{21}=k_{2} d+v_{0} g \\
& t_{22}=k_{2} e+v_{0} h \\
& t_{23}=k_{2} f+v_{0} i \\
& t_{24}=k_{2} q+v_{0} r \\
& t_{31}=g \\
& t_{32}=h \\
& t_{33}=i \\
& t_{34}=r
\end{aligned}
$$

So, given the coordinates of o target spot and measuring the coordinates of this spot as projected on the image plane, the elements of [T] that connect the coordinates can be determined. Knowing the elements of [i], the position and orientation of the camera relative to target $\left(\mathrm{X}_{s}, \mathrm{Y}_{\mathbf{s}}, \mathrm{Z}_{\mathrm{s}}\right.$ and 
$1, m, n)$ can be found.

This problem can be somewhat simplified when some information is known about the camera parameters $\left(\mathrm{k}_{1}\right.$ and $\mathrm{k}_{2}$ or $\mathrm{u}_{0}$ and $\left.\mathrm{v}_{0}\right)$. If four target spots are used and the spots are placed in general position on a plane (let that plane be the plane where $z=0$ ) then the general approach can be written as :

$$
\left[\begin{array}{c}
u_{i} \\
v_{i} \\
1
\end{array}\right]=\left[\begin{array}{llll}
t_{11} & t_{12} & t_{13} & t_{14} \\
t_{21} & t_{22} & t_{23} & t_{24} \\
t_{31} & t_{32} & t_{33} & t_{34}
\end{array}\right]\left[\begin{array}{c}
x_{i} \\
y_{i} \\
0 \\
1
\end{array}\right]
$$

where $i=1,2,3,4$

This leads to:

$$
\left[\begin{array}{l}
u_{i} \\
v_{i} \\
1
\end{array}\right]=\left[\begin{array}{lll}
t_{11} & t_{12} & t_{14} \\
t_{22} & t_{22} & t_{24} \\
t_{31} & t_{32} & t_{34}
\end{array}\right]\left[\begin{array}{c}
x_{i} \\
y_{i} \\
1
\end{array}\right]
$$

Taking advantage of the use of a homogeneous coordinate system (dividing each element by the last element, r) the above matrix elements can be written as: 


$$
\begin{aligned}
& t_{11}=\left(k_{1} a+u_{0} g\right) / r \\
& t_{12}=\left(k_{1} b+u_{0} h\right) / r \\
& t_{14}=\left(k_{1} p+u_{0} r\right) / r \\
& t_{21}=\left(k_{2} d+v_{0} g\right) / r \\
& t_{22}=\left(k_{2} e+v_{0} h\right) / r \\
& t_{24}=\left(k_{2} q+v_{0} r\right) / r \\
& t_{31}=g / r \\
& t_{32}=h / r \\
& t_{34}=1
\end{aligned}
$$

Knowing $\mathrm{x}_{i}$ and $\mathrm{y}_{i}$, and measuring $\mathrm{u}_{i}$ and $\mathrm{v}_{i}$, the elements of [T] can be found. Then, these values, $k_{1}$ and $k_{2}$, and the system constraint equations can be used to determine the values of $a, b, c, d, e, f, g, h$, and $i . p, q$, and $r$ can then be found. Once this is done, $X_{s}, Y_{s}, Z_{s}, 1, m$, and $n$ can be computed.

Because the rotation matrix, [R], is a pure orthonormal matrix, $[R]^{-1}$ is the same as $[R]^{T}$ and the determinant of [R] is equal to 1 [Kanattani, 1987]. These conditions give the following constraints on the elements of [R]:

$$
\begin{aligned}
& a^{2}+b^{2}+c^{2}=1 \\
& d^{2}+e^{2}+f^{2}=1 \\
& g^{2}+h^{2}+i^{2}=1
\end{aligned}
$$




$$
\begin{aligned}
& a=e i-h f \\
& b=f g-d i \\
& c=d h-e g \\
& d=h c-b i \\
& e=a i-g c \\
& f=b g-a h \\
& g=b f-e c \\
& h=c d-a f \\
& i=a e-b d \\
& a d+b e+c f=0 \\
& d g+e h+f i=0 \\
& a g+b h+c i=0 \\
& \text { Once the elements of [T] are known, the unknowns are }
\end{aligned}
$$
found as follows:

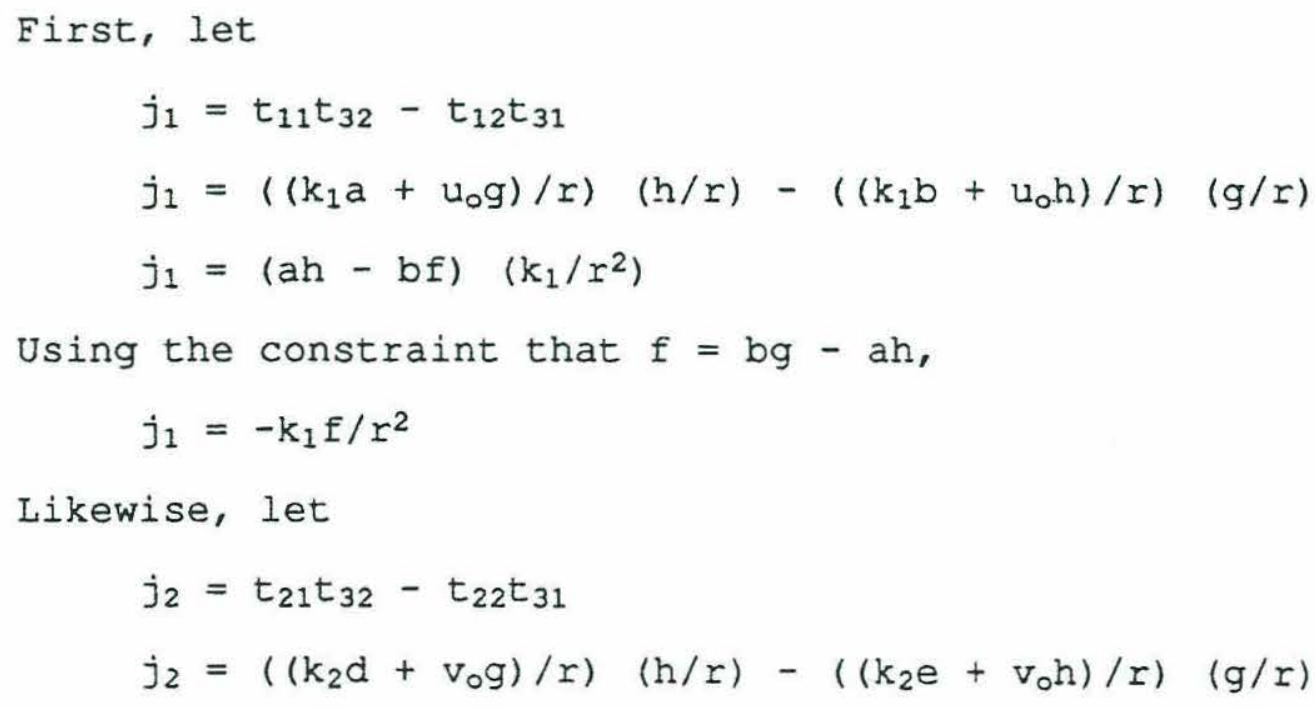




$$
j_{2}=(h d-e g)\left(k_{2} / r^{2}\right)
$$

Using the constraint that $c=$ hd - eg,

$$
j_{2}=k_{2} c / r^{2}
$$

Remember that since $t_{i j}$ are known, $j_{1}$ and $j_{2}$ are known.

Also,

$$
\left(t_{31}\right)^{2}+\left(t_{32}\right)^{2}=(g / r)^{2}+(h / r)^{2}=\left(g^{2}+h^{2}\right) / r^{2}
$$

Expressing the constraints as,

$$
\begin{aligned}
& g^{2}+h^{2}+i^{2}=c^{2}+f^{2}+i^{2}=1 \\
& \text { gives, } \\
& g^{2}+h^{2}=c^{2}+f^{2}
\end{aligned}
$$

Substituting this into the expression for

$$
\left(t_{31}\right)^{2}+\left(t_{32}\right)^{2}
$$

gives:

$$
\left(t_{31}\right)^{2}+\left(t_{32}\right)^{2}=\left(f^{2}+c^{2}\right) / r^{2}=r^{2}\left(f^{2}+c^{2}\right) / r^{4}
$$

Now substitute for $f$ and $c$ values using $j_{1}$ and $j_{2}$ :

$$
\left(t_{31}\right)^{2}+\left(t_{32}\right)^{2}=\left(\left(j_{1} / k_{1}\right)^{2}+\left(j_{2} / k_{2}\right)^{2}\right) r^{2}
$$

Because $t_{31}, t_{32}, j_{1}, j_{2}, k_{1}$, and $k_{2}$ are known, this equation can be solved for $r^{2}$ and $r$ (assume that $r$ is positive if the image is in front of the camera).

Then, since $f^{2}+c^{2}=1-i^{2}$, substitute and solve $\left(t_{31}\right)^{2}+\left(t_{32}\right)^{2}=\left(1-i^{2}\right) / r^{2}$ for $i^{2}$.

$i$ will be positive if the cross product of the $U$ and $V$ axes is in the same direction as the $Z$ axis and negative if in 
the opposite direction. This will depend on the way that the system coordinate system is initially established.

Now, knowing $r$ and $i$, other values follow quickly:

$$
\begin{aligned}
& t_{31}=g / r, \quad g=t_{31} r \\
& t_{32}=h / r, \quad h=t_{32} r \\
& j_{1}=k_{1} f / r^{2}, \quad f=j_{1} r^{2} / k_{1} \\
& j_{2}=k_{2} c / r^{2}, \quad c=j_{2} r^{2} / k_{2}
\end{aligned}
$$

Next, look at

$$
\begin{aligned}
t_{11} t_{31}+t_{12} t_{32}= & \left(\left(k_{1} a+u_{\circ} g\right) / r\right)(g / r)+ \\
& \left(\left(k_{1} b+u_{\circ} h\right) / r\right)(h / r) \\
= & \left(k_{1} / r^{2}\right)(a g+b h)+ \\
& \left(u_{0} / r^{2}\right)\left(g^{2}+h^{2}\right)
\end{aligned}
$$

From the constraint, ag $+\mathrm{bh}+\mathrm{ci}=0$, make the substitution:

$$
a g+b h=-c i
$$

Then, $t_{11} t_{31}+t_{12} t_{32}=-\left(k_{1} c i / r^{2}\right)+u_{0}\left(g^{2}+h^{2}\right) / r^{2}$

$$
t_{11} t_{31}+t_{12} t_{32}+k_{1} c i / r^{2}=u_{0}\left(g^{2}+h^{2}\right) / r^{2}
$$

and $u_{0}$ can be determined:

$$
\begin{aligned}
& u_{\circ}=\left(r^{2} /\left(g^{2}+h^{2}\right)\right)\left(t_{11} t_{31}+t_{12} t_{32}+k_{1} c i / r^{2}\right) \\
& \text { Now, look at } \\
& t_{21} t_{31}+t_{22} t_{32}=\left(\left(k_{2} d+v_{0} g\right) / r\right)(g / r)+ \\
& \quad\left(\left(k_{2} e+v_{0} h\right) / r\right)(h / r)
\end{aligned}
$$




$$
\begin{array}{r}
=\left(k_{2} / r^{2}\right)(d g+e h)+ \\
\left(v_{0} / r^{2}\right)\left(g^{2}+h^{2}\right)
\end{array}
$$

From the constraint, $d g+e h+f i=0$, make the substitution:

$$
\begin{aligned}
d g+e h= & -f i \\
\text { Then, } t_{21} t_{31}+t_{22} t_{32}=- & \left(k_{2} f i / r^{2}\right)+ \\
& \left(v_{0} / r^{2}\right)\left(g^{2}+h^{2}\right)
\end{aligned}
$$

and $\mathrm{v}_{\circ}$ can be determined:

$v_{0}=\left(r^{2} /\left(g^{2}+h^{2}\right)\right)\left(t_{21} t_{31}+t_{22} t_{32}+k_{2} f i / r^{2}\right)$

Now, solve for $a, b, p, d, e$, and $q$ as follows:

since $t_{11}=\left(k_{1} a+u_{0} g\right) / r$,

$a=\left(r t_{11}-u_{0} g\right) / k_{1}$

since $t_{12}=\left(k_{1} b+u_{0} h\right) / r$,

$\mathrm{b}=\left(r \mathrm{t}_{12}-\mathrm{u}_{\mathrm{o}} \mathrm{h}\right) / \mathrm{k}_{1}$

since $t_{14}=\left(k_{1} p+u_{0} r\right) / r$,

$p=\left(r / k_{1}\right)\left(t_{14}-u_{0}\right)$

since $t_{21}=\left(k_{2} d+v_{0} g\right) / r$,

$d=\left(r t_{21}-v_{\circ} g\right) / k_{2}$

since $t_{22}=\left(k_{2} e+v_{0} h\right) / r$,

$e=\left(r t_{22}-v_{0} h\right) / k_{2}$

since $t_{24}=\left(k_{2} q+v_{0} r\right) / r$,

$q=\left(r / k_{2}\right)\left(t_{24}-v_{0}\right)$

Because $a, b, c, d, e, f, g, h, i, p, q$, and $r$ are now 
known, it is possible to use them to determine $\mathrm{X}_{\mathbf{s}}, \mathrm{Y}_{\mathbf{s}}, \mathrm{Z}_{\mathbf{s}}, \mathrm{I}$, $m$, and $n$.

To solve for $X_{s}, Y_{s}$, and $Z_{s}$ solve equations:

$x_{s}=-a p-d q-g r$

$Y_{s}=-b p-e q-h r$

$z_{s}=-c p-f q-i r$

To find $1, m$, and $n$ first use $f=\sin m$ and solve for $m$ then use this value in the equations for $e$ and $i$ to obtain 1 and $n$ :

$$
\begin{aligned}
& m=\sin ^{-1}(f) \\
& 1=\cos ^{-1}(e / \cos (m)) \\
& n=\cos ^{-1}(i / \cos (m))
\end{aligned}
$$

The above presentation shows that by knowing the focal length and scaling of the camera, one can find the position and orientation of the camera if the target has four features that can be identified and located. Other methods of determining camera location solve the general approach by identifying more target spots and using least squares methods of getting a best fit that satisfies the transformation equations and the constraint equations [Fischler, 1980], [Huang, 1981], [Lowe, 1980], [Sutherland, 1974].

The method described in this paper relies heavily on accurate measurements of $u_{i}$ and $v_{i}$. It is generally more 
zccurate to use $k_{1}$ and $k_{2}$ as known values (vice $u_{0}$ ard $v_{0}$ ) in solving the problem because $k_{1}$ and $k_{2}$ are typically more accurately found when calibrating the camera. Because this solution method is not exact, the results may need some conditioning if the rueasurements of $u_{i}$ and $v_{i}$ are not accurate enough for the desired application. However, this closed form solution to the problem has the advantage of using a simple target design and requiring little computing power to obtain the solution.

of course, using this method depends on the ability to find the elements of [T]. A description of how these elements can be found follows.

\subsection{Determining the Transformation Matrix}

The coordinates in the above matrices have been expressed using homogeneous coordinate systems. In a homogeneous coordinate system, the three dimensional point $\mathrm{x}, \mathrm{y}, \mathrm{z}$ is represented by the four-tuple wx,wy,wz,w and the two dimensional point $u, v$ is represented by the three-tuple wu,wv,w. After using the homogeneous coordinates, the three and two dimensional coordinates are obtained by dividing all components by the last component, w. So, although the perspective transformation has been written as: 


$$
\left[\begin{array}{l}
u_{i} \\
v_{i} \\
1
\end{array}\right]=\left[\begin{array}{lll}
t_{11} & t_{12} & t_{14} \\
t_{21} & t_{22} & t_{24} \\
t_{31} & t_{32} & 1
\end{array}\right]\left[\begin{array}{c}
x_{i} \\
y_{i} \\
1
\end{array}\right]
$$

in practice, after performing the matrix multiplication, the first two rows are divided by the third. This gives the following expressions for $u_{1}$ and $v_{i}$ :

$$
\begin{aligned}
& u_{i}=\left(t_{11 x_{i}}+t_{12 Y_{i}}+t_{14}\right) /\left(t_{31} x_{i}+t_{32 Y_{i}}+1\right) \\
& v_{i}=\left(t_{21} x_{i}+t_{22 Y_{i}}+t_{24}\right) /\left(t_{31} x_{i}+t_{32 Y_{i}}+1\right)
\end{aligned}
$$

where $i=1,2,3,4$

This yields eight equations to find the eight unknowns $t_{11}, t_{12}, t_{14}, t_{21}, t_{22}, t_{24}, t_{31}, t_{32}:$

$u_{1}+u_{1} x_{1} t_{31}+u_{1} y_{1} t_{32}-x_{1} t_{11}-y_{1} t_{12}-t_{14}=0$

$u_{2}+u_{2} x_{2} t_{31}+u_{2} y_{2} t_{32}-x_{2} t_{11}-y_{2} t_{12}-t_{14}=0$

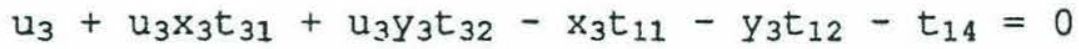

$u_{4}+u_{4} x_{4} t_{31}+u_{4} y_{4} t_{32}-x_{4} t_{11}-y_{4} t_{12}-t_{14}=0$

$v_{1}+v_{1} x_{1} t_{31}+v_{1} y_{1} t_{32}-x_{1} t_{21}-y_{1} t_{22}-t_{24}=0$

$v_{2}+v_{2} x_{2} t_{31}+v_{2} y_{2} t_{32}-x_{2} t_{21}-y_{2} t_{22}-t_{24}=0$

$v_{3}+v_{3} x_{3} t_{31}+v_{3} Y_{3} t_{32}-x_{3} t_{21}-y_{3} t_{22}-t_{24}=0$

$v_{4}+v_{4} x_{4} t_{31}+v_{4} y_{4} t_{32}-x_{4} t_{21}-y_{4} t_{22}-t_{24}=0$

The above equations can be written in matrix form as: 
$-\left[\begin{array}{l}u_{1} \\ u_{2} \\ u_{3} \\ u_{4} \\ v_{1} \\ v_{2} \\ v_{3} \\ v_{4}\end{array}\right]=\left[\begin{array}{cccccccc}-x_{1} & -y_{1} & -1 & 0 & 0 & 0 & x_{1} u_{1} & y_{1} u_{1} \\ -x_{2} & -y_{2} & -1 & 0 & 0 & 0 & x_{2} u_{2} & y_{2} u_{2} \\ -x_{3} & -y_{3} & -1 & 0 & 0 & 0 & x_{3} u_{3} & y_{3} u_{3} \\ -x_{4} & -y_{4} & -1 & 0 & 0 & 0 & x_{4} u_{4} & y_{4} u_{4} \\ 0 & 0 & 0 & -x_{1} & -y_{1} & -1 & x_{1} v_{1} & y_{1} v_{1} \\ 0 & 0 & 0 & -x_{2} & -y_{2} & -1 & x_{2} v_{2} & y_{2} v_{2} \\ 0 & 0 & 0 & -x_{3} & -y_{3} & -1 & x_{3} v_{3} & y_{3} v_{3} \\ 0 & 0 & 0 & -x_{4} & -y_{1} & -1 & x_{4} v_{4} & y_{4} v_{4}\end{array}\right]\left[\begin{array}{l}t_{11} \\ t_{12} \\ t_{14} \\ t_{21} \\ t_{22} \\ t_{24} \\ t_{31} \\ t_{32}\end{array}\right]$ 
Chapter 3 Use of Prepared, Passive Targets

\subsection{Target Design Criteria}

Several methods have been developed to determine the position of a camera and track the motion of a camera relative to a landmark or target. Some methods involve "teaching" the tracking system some general shapes it can identify and then determining its position relative to the shapes by use of stereo vision or some other method [Nasr, 1988], [Sugihara, 1988]. A second approach is to construct a target of spots and, knowing the location of the spots on the target, use perspective transformation analysis to determine camera position.

All of the applications discussed in this paper involve operating and controlling an ROV in an underwater environmment. The terrain and possible landmarks are varied depending on the location and depth. In fact, in many cases, the shapes present may not be known (if they exist at all) until the ROV is at the site. For this reason, "teaching" the tracking system some general shapes or landmarks may be impractical (except in a limited number of applications). Also, the algorithms used for determining camera position relative to landmarks are typically numerically based and 
involve either long computing times or large amounts of computer memory space.

A simpler, more direct method uses perspective transformations to orient and position a target consisting of a known pattern of points. As explained in Chapter 2, a closed form solution of the problem is obtained by using four target spots and information about the camera. Once the actuil spot coordinates and the image plane spot coordinates are known, the transformation matrix can be found and its elements used to solve for the camera position and orientation.

Given that a prepared target will be used, it must now be decided whether the target will be "active" or "passive". An active target uses a power source to illuminate the target spots so that the camera can detect them. For example, some tracking systems use infrared light emitting diodes as the target spots. These IRED spots act as beacons to the camera; the camera detects the location of the infrared light and the transformation matrix can be determined. Passive targets use ambient or reflected light to illuminate the spots. For example, the target spots can be made of a reflective material and light from lights or a strobe will be reflected from the spots into the camera lens where the image plane spot 
coordinates can be determined.

For underwater applications, where light is absorbed over short distances and where it is difficult to keep a target powered for long periods of time, an active target may be impractical. Whereas the JASON ROV is powered from shipboard generators via the ARGO, an active target would require its own power supply or a link to the ship. Any battery powered active target would need occassional battery replacement and that replacement might be needed at very inconvenient times. An active target, once activated, would emit its signal even if the camera were too far away to detect the signal and so the signal (and its power) would be wasted. A passive target would not require any type of power supply and would send out a signal only if the camera were close enough to illuminate the target. For these reasons, a passive target was designed and used in the measurements presented in this paper.

The target used in the optical tracking system for JASON was composed of four reflective spots. The spots were placed in general position on a flat $(z=0)$, non-reflective surface. The $x, y$ coordinates of these spots make up the $x_{1}, y_{1}$ matrix used in Chapter 2 to determine the transformation matrix. In the solution method, these values will not change. As one of JASON's video cameras is pointed toward the 
target, the light from the camera light reflects off the spots and into the camera lens where the image of the target is projected onto the image plane. The ISCAN video tracking system detects the location of the target spots as they appear on the image plane. These values give the $u_{i}, v_{i}$ matrix of the solution method. As JASON moves toward and away from the target and as JASON changes its pitch, roll, and yaw, the elements of the $u_{i}, v_{i}$ matrix change. Solving for the new transformation matrix that is produced each time JASON changes its position and orientation gives the current- values of $\mathrm{X}_{s}, \mathrm{Y}_{s}, \mathrm{Z}_{s}$ and $\mathrm{I}, \mathrm{m}, \mathrm{n}$. These position and oriertation values can then be used in the JASON control functions to navigate, keep station, or dock.

\subsection{Target Spot Placement}

The target spots need to be placed on the target so that they will be inside the camera field of view at the point of closest approach. The spots need also to be placed far enough apart so that they are distinguishable from each other at long ranges. They must be far enough away from each other that the spot tracking system can detect each spot individually.

If we assume that the Rov will be .25 meters away from the target at the point of closest approach, the target spots 
should fill the image plane (this will give the maiximum spot spread at long range). Looking straight at the target at this point of closest approach, the maximum spread of the spotz can be determined (this will also depend on the camera focal length).

Because the spots are positioned generally, (nonsymmetric) this allows the spots to be placed near the edges of this window. Figure 8 gives a possible spot positioning. 
Chapter 4 Description of the ISCAN Video Tracking System

The ISCAN RK-446 Video Tracking System is a real time digital image processor that tracks and measures contrasting targets in the field of view of a video camera. The system determines the center of a target spot to within one-half pixel giving the horizontal and vertical coordinates of the target spot as they appear on the image plane of the camera. The system updates the horizontal and vertical position measurements every $16 \mathrm{msec}$ (at a frequency of $60 \mathrm{~Hz}$ ) and gives this information in a computer compatible, parallel format. The image processor uses an artifact elimination algorithm that rejects noise or clutter. The RK-446 divides the image plane into a grid of 511 pixels horizontally and 255 pixels vertically. 


\section{Chapter 5 Testing}

\subsection{Test Design}

To test the above described analytical method, a test target of four spots in general position was designed. The target spots were precision placed and drilled to be within one mil (within one thousandth of an inch). The target spots were placed so that the target $Y$ coordinate axis was in the middle of the target plate and the $\mathrm{x}$ coordinate axis was $5 \mathrm{~cm}$ above the base plate. This positioning gives the $x_{i}$ and $y_{i}$ values given in appendix A.

The target plate was placed perpendicular to the base plate. The base plate was made of plexiglass and had five holes drilled at the locations shown in figure 9. Pegs of various heights were placed in the holes and the camera was then sighted along these so that a known camera orientation could be determined. For example, by placing pegs $5 \mathrm{~cm}$ high into holes $A$ and $B$ and sighting along these pegs, the camera was at a height of $\mathrm{Y}=0$ and at a pan angle of $0^{\circ}$ (relative to the target coordinate system). Pegs in holes $\mathrm{A}$ and $\mathrm{C}$ gave $\mathrm{a}$ pan angle of $30^{\circ}$. Pegs in holes $A$ and $D$ gave a pan angle of $45^{\circ}$. Pegs in holes $A$ and $E$ gave a pan angle of $60^{\circ}$. By placing a $10 \mathrm{~cm}$ peg in hole B, a tilt angle of $25.7^{\circ}$ was 
obtained. A $14 \mathrm{~cm}$ peg in hole B gave a tilt angle of $40.87^{\circ}$.

The target was placed on a table over which calibrated paper was placed. Using the RK-446 Video Tracking System, the values of $u_{i}$ and $v_{i}$ were recorded as the camera was placed at various positions and orientations. The coordinates and results of these measurements are listed in the appendices.

\subsection{Camera Calibration}

Prior to complete testing, it is necessary to calibrate the camera so that the horizontal and vertical scaling factors, $k_{1}$ and $k_{2}$ can be determined.

The camera was calibrated by placing the four target spots in two parallel planes. This gave eight image plane spot measurements. The target was placed in front of the camera and the image plane coordinates of the target spots were recorded. The target was then moved a known distance along the $z$ axis. At the next target location, the image plane spot coordinates were again recorded. Knowing the $x_{i}, y_{i}, z_{i}$ coordinates of the target spots and the $u_{i}, v_{i}$ measurements of the eight spots gave a total of sixteen equations to find the eleven unknowns of the tranformation matrix (elements $t_{11}$ through $t_{34}$ ). A least-squares approach was used to find the transformation matrix elements. Once these elements were 
known, the system constraint equations were used to solve for $k_{1}$ and $k_{2}$. (In much the same way as described in Chapter 2 but by using the full transformation matrix since no camera parameters were yet known.)

This calibration was done for the three lenses to be tested: a $4.8 \mathrm{~mm}$ focal length lens, an $8 \mathrm{~mm}$ focal length lens, and a $12 \mathrm{~mm}$ focal length lens. The calibration results for $\mathrm{k}_{1}$ and $k_{2}$ for each lens are given in appendices $A, B$, and C respectively.

\subsection{Test Results}

The first system tests were performed using the test target and a Sony DXC-101 ccd video camera in air. The target spot coordinates, known camera location and orientation, image plane measurements, and algorithm results are listed in appendices A, B, and C for the $4.8 \mathrm{~mm}, 8 \mathrm{~mm}$, and $12 \mathrm{~mm}$ lenses respectively.

There are several sources of possible error. First, the solution method assumes that the camera behaves as a pin-hole camera. The $4.8 \mathrm{~mm}$ lens is probably too short for this to be true. For the longer lenses, the error due to non-pin-hole behavior is inconsequential. Errors due to imperfect calibration are also possible (this is probably why the $8 \mathrm{~mm}$ 
lens results are not as good as those of the $12 \mathrm{~mm}$ lens). Third, the ISCAN Video Tracking System is accurate to within one-half pixel when finding the target spot centers. Any error in finding the spot centers would result in incorrect image plane coordinate measurements. The method seems to be largely unaffected by such errors when the longer focal length lenses are used. Inputting half pixel changes in the measurements did slightly change the $X_{s}$ and/or $Y_{s}$ results but had a smaller affect on the $z_{s}$ result. However, doing the same for the $4.8 \mathrm{~mm}$ lens had dramatic effects. For example, a non-symmetric half-pixel change in the $u_{i}$ and $v_{i}$ measurements changed the $X_{s}$ and/or $Y_{3}$ results by as much as $50 \%$ (but again had little affect on the $z_{s}$ result). This is probably due to the less pin-hole behavior of the $4.8 \mathrm{~mm}$ lens. The consistently largest and most important source of error for the system, independent of the lens used, results when the system in used underwater and is subjected to the effects of refraction. 


\section{Chapter 6 Underwater Effects}

To this point, all analysis and experimentation has involved the camera, target, and tracking system in an air environment. When the system is placed in the water, optical effects can dramatically affect the system's performance.

For the target, the underwater environment creates no difficulties. The reflectance of the spots will be the same as in air and will not change with depth.

For the camera, there are the problems of turbulence, suspended particles, and refraction.

Turbulence in the water gives rise to inhomogeneities along the optical paths of the camera. These patches of water have different optical properties than surrounding water and cause optical distortion. However, these effects are small and are generally a factor only in long range, high resolution applications.

Suspended particles scatter the light and create a type of veil between the target and the camera. This veil of scattered light reduces the contrast between the light and dark of the target. However, by designing target spots that are initially in sharp contrast with the target plate, this effect can be ignored. 
Refraction is the bending of a ray of light as it passes through materials of different optical properties. The cameras on JASON are housed inside cases that have a flat window through which the cameras look. This gives three interfaces: the water/window interface, the window/air interface, and the air/lens interface. This system can be modelled as consisting of two components, the window producing an image and the air/lens focusing that image onto the image plane. Because the index of refraction in water is approximately 1.33 times greater than the index of refraction in air, the distance from the lens to the target along the line of sight will appear to be 1.33 times shorter than it really is. Also, because of the bending of the light rays at the air/lens interface, lateral distances will seem different than they actually are. For example, a horizontal field of view in air of $77.3^{\circ}$ will appear as a $56^{\circ}$ field of view in water. When the camera looks at the target and the image piane coordinates of the target spots are measured, they will appear farther apart than they should be.

Because the decreased field of view and the shortening of length along the line of sight are independent of distance, it should be possible to implement corrections in the algorithm.

It is worth mentioning that the water index of refraction 
is a function of depth, salinity, and turbidity and can change by as much as two percent. Because the cameras used in determining position and orientation have fixed focal lengths, this variability in the index of refraction may affect system accuracy (by slightly shifting the focal length). However, since the index of refraction error is less than the system error, it is probably not necessary to account for these changes in the algorithm.

In using perspective transformations, there are two options to pursue.

First, because refraction moves the image plane spots farther apart than they would appear in air, the spots can be scaled back to where they would appear without refraction. However, the distance the spots should be scaled depends on the incident angle of the light which, in turn, depends on the distance to the target. To be completely accurate, the $u_{i}$ and $v_{i}$ measurements should be scaled by 1.33 (the ratio of the index of refraction in water and the index of refraction in air) and the perspective transformation performed. The factor of 1.33 should then be re-evaluated by using the computed distance to the target. This new factor (slightly larger than 1.33 ) should then be used to re-scale the $u_{i}$ and $v_{i}$ measurements and a new perspective transformation should 
be performed. Using the newly computed distance to the target, the factor of 1.33 should again be revised and the process repeated until the result settles on the actual values.

However, this process takes us away from our goal of avoiding any time consuming numerical methods (like iteration). By analyzing the refraction geometry, one finds that if the $u_{i}$ and $v_{i}$ measurements are scaled by 1.33 and the perspective transformation performed, the result will be within allowable error domains. For the test target, doing a one-time scaling of 1.33 introduces an error of less than three percent for an $8 \mathrm{~mm}$ lens and an error of less than one percent for a $12 \mathrm{~mm}$ lens. For a $4.8 \mathrm{~mm}$ lens, the error is larger and the scaling introduces the instability previously described.

A second method of correcting for water effects is to use a corrective dome as the window on the camera housing. A corrective dome is designed for a specific focal length lens and a specific camera housing. The dome corrects the refraction so that the image appears as it would in air. However, corrective domes tend to shrink and slide in high pressure environments (which causes some image distortion) and can be quite expensive to produce. In general, corrective 
domes work well for wide angle lenses (air fields of view greater than eighty degrees) and would be the best approach for the $4.8 \mathrm{~mm}$ lens. 


\section{Chapter 7 Conclusions}

The method described in this paper seems to work well for camera lenses with focal lengths of at least $8 \mathrm{~mm}$. Underwater effects can, to within an acceptable degree of error, be compensated for by scaling the $u_{i}$ and $v_{i}$ measurements toward the image plane center by a factor of 1.33 .

The method does not work as well for shorter focal length lenses $(4.8 \mathrm{~mm}$ in particular) although the computed range values are reasonable and usable. Errors introduced by using a $4.8 \mathrm{~mm}$ lens will be increased when underwater but can be improved to in-air values by using a compensating dome as the window on the camera housing.

Errors in the $u_{i}$ and $v_{i}$ measurements affect the $X_{3}, Y_{s}$, and orientation calculations but have a much smaller effect on the $Z_{s}$ calculation.

The method is non-numerical and should be easy to implement. The U,V measurements from the ISCAN Video Tracking System can be output to a JASON computer (via a parallel I/O interface) where real-time ROV position can be calculated. This position can then be used in JASON control functions. 


\section{Low Frequency Long Baseline Narrow Band Pulses $\sim 10 \mathrm{kHz}$}

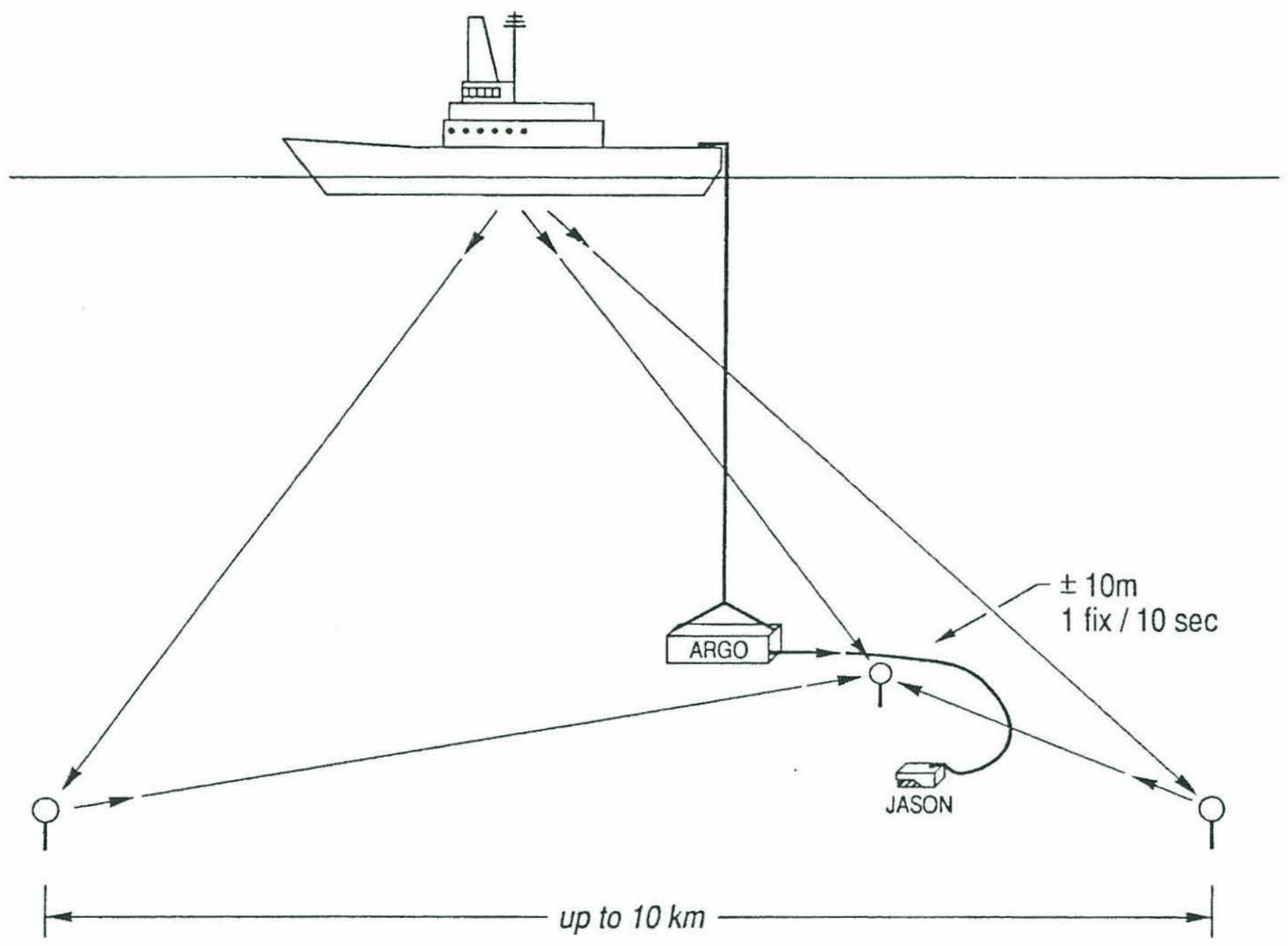

ARGO/JASCN Navigation Referenced to Ship

Figure 1 
High Frequency, Relative to ARGO

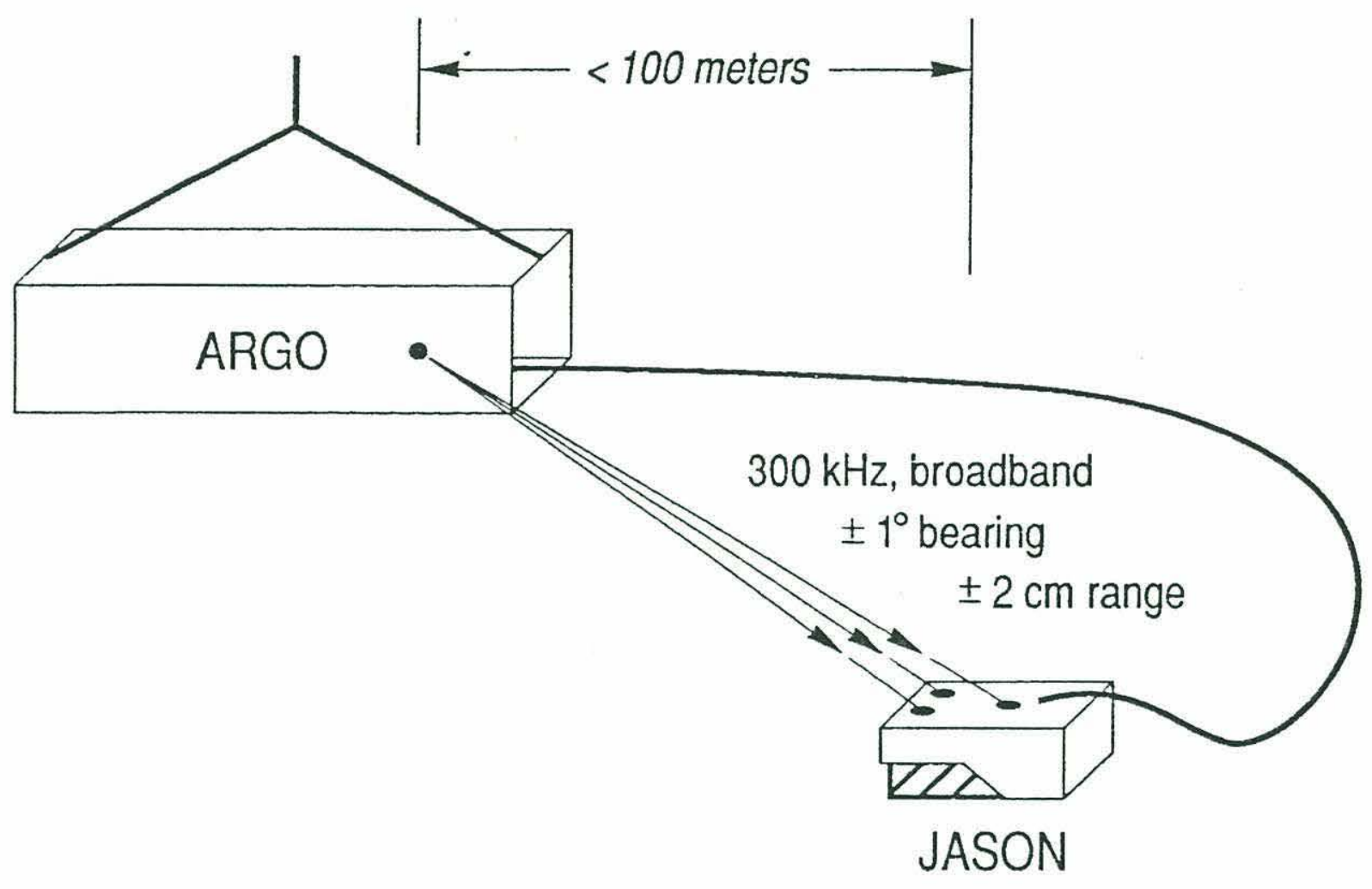

JASON Navigation Referenced to ARGO

Eigure 2 


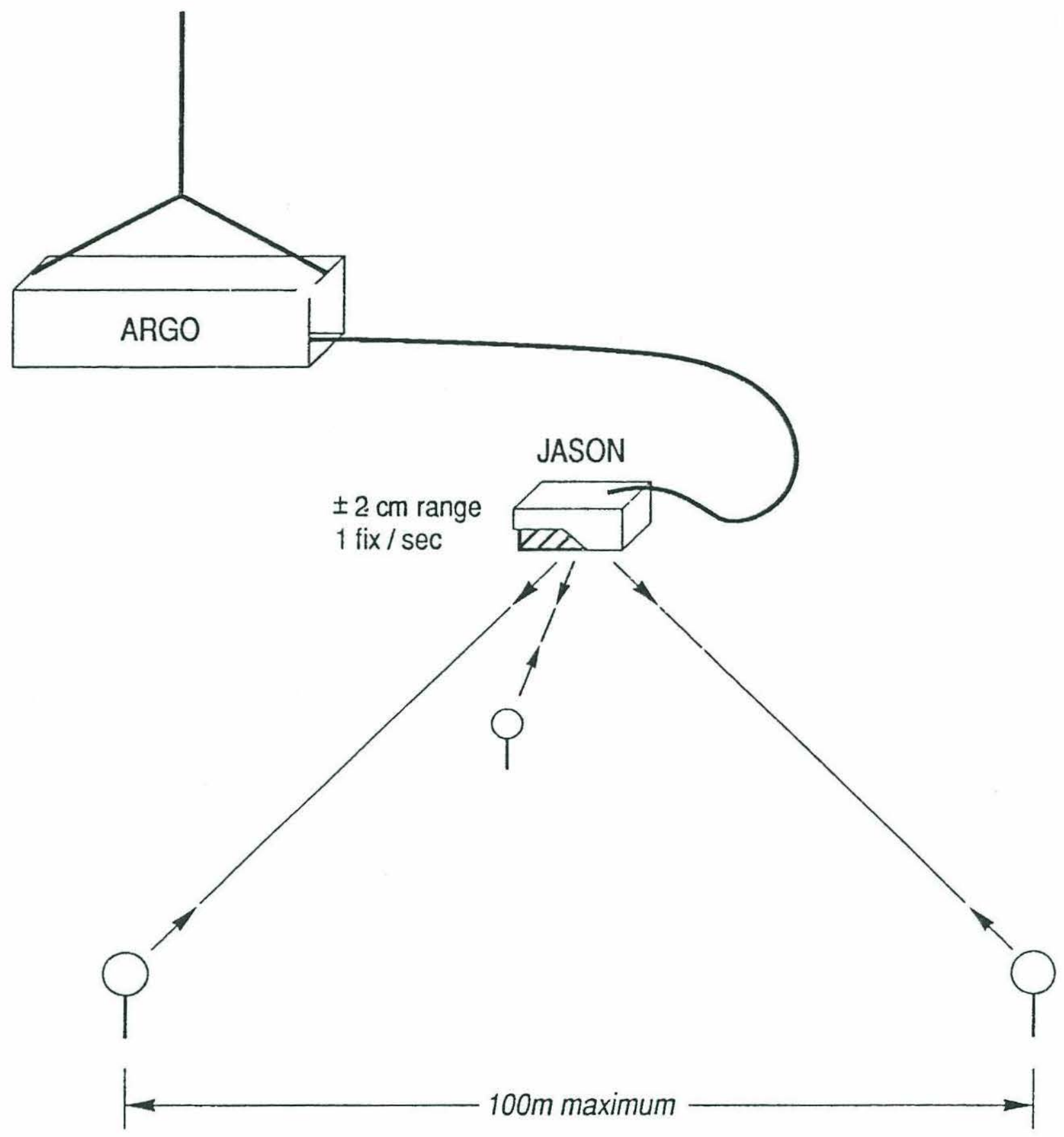

JASON Navigation Referenced to Seafioor

Eigure 3 


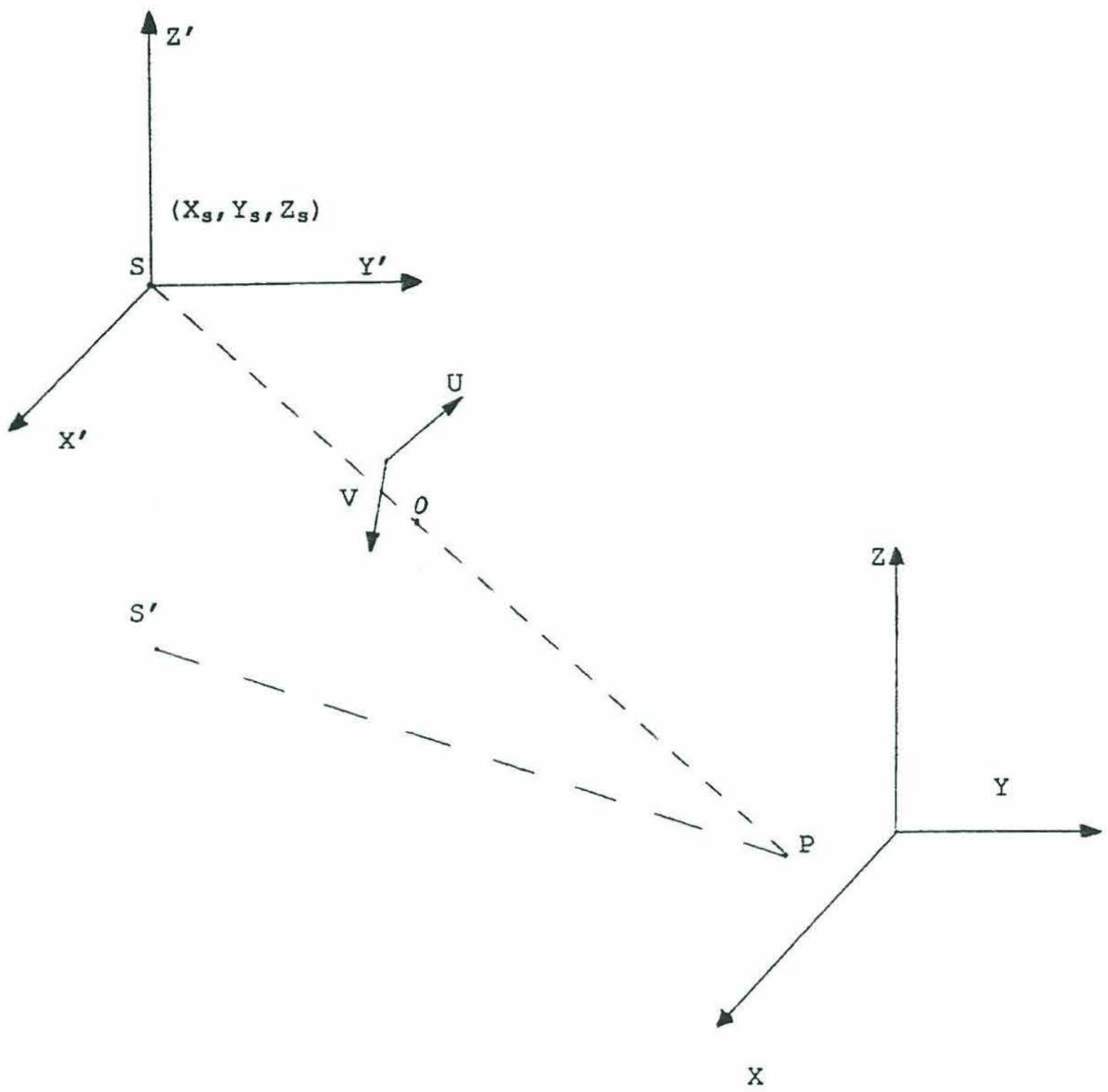

Displacement of Camera Coordinate system

Figure 4 

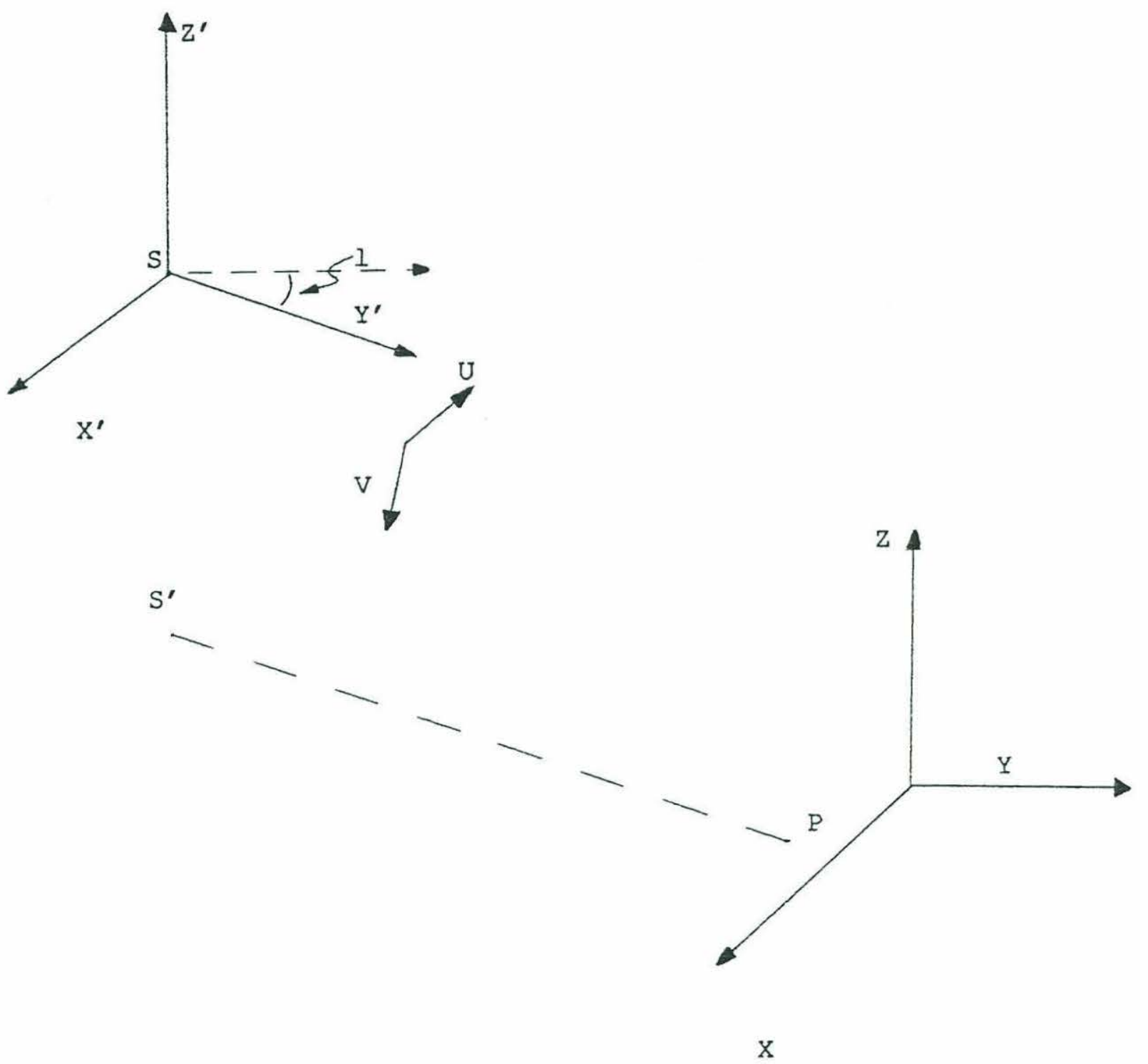

Pan Angle Rotation

Eigure 5 


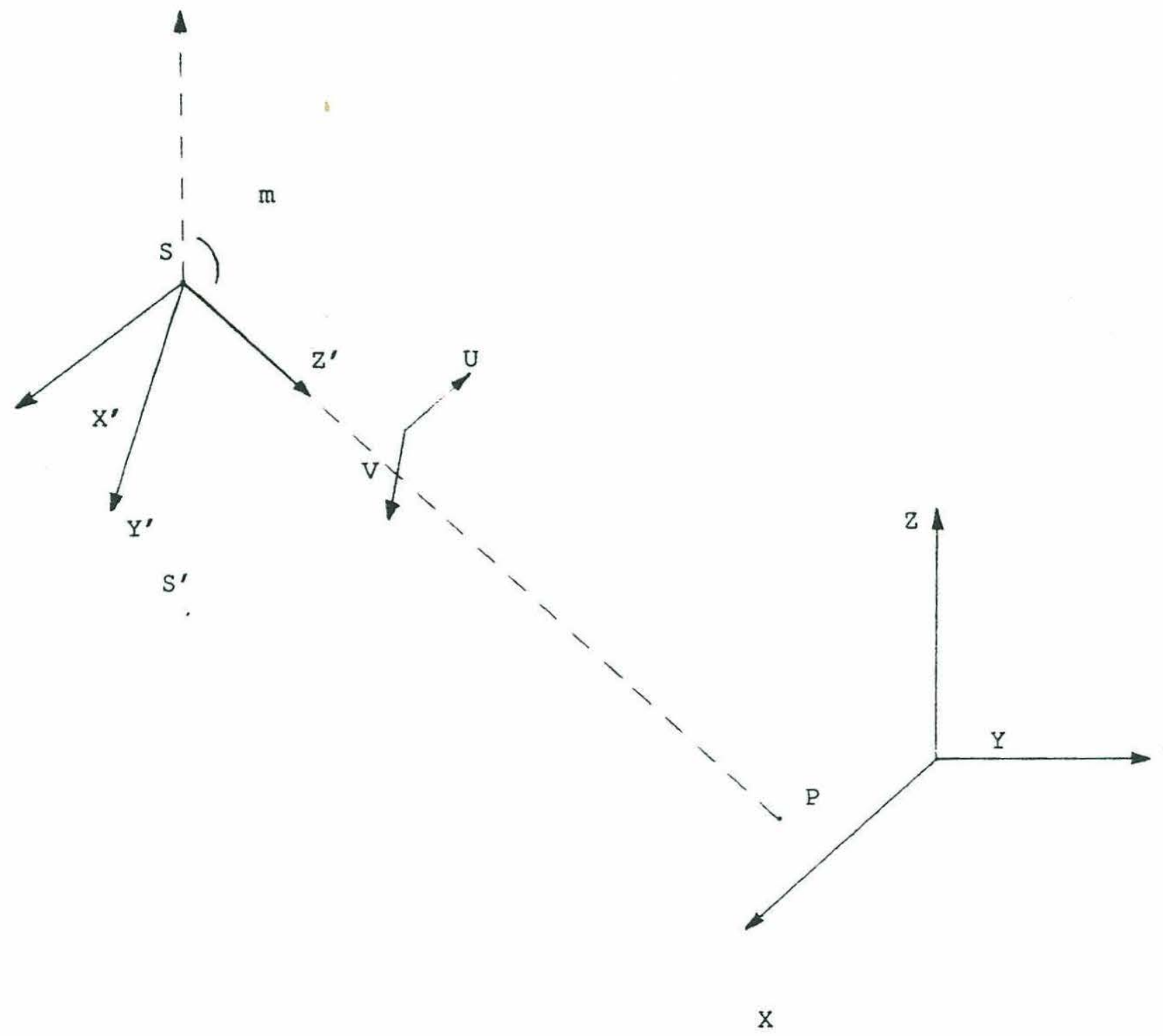

Tilt Angle Rotation

Figure 6 

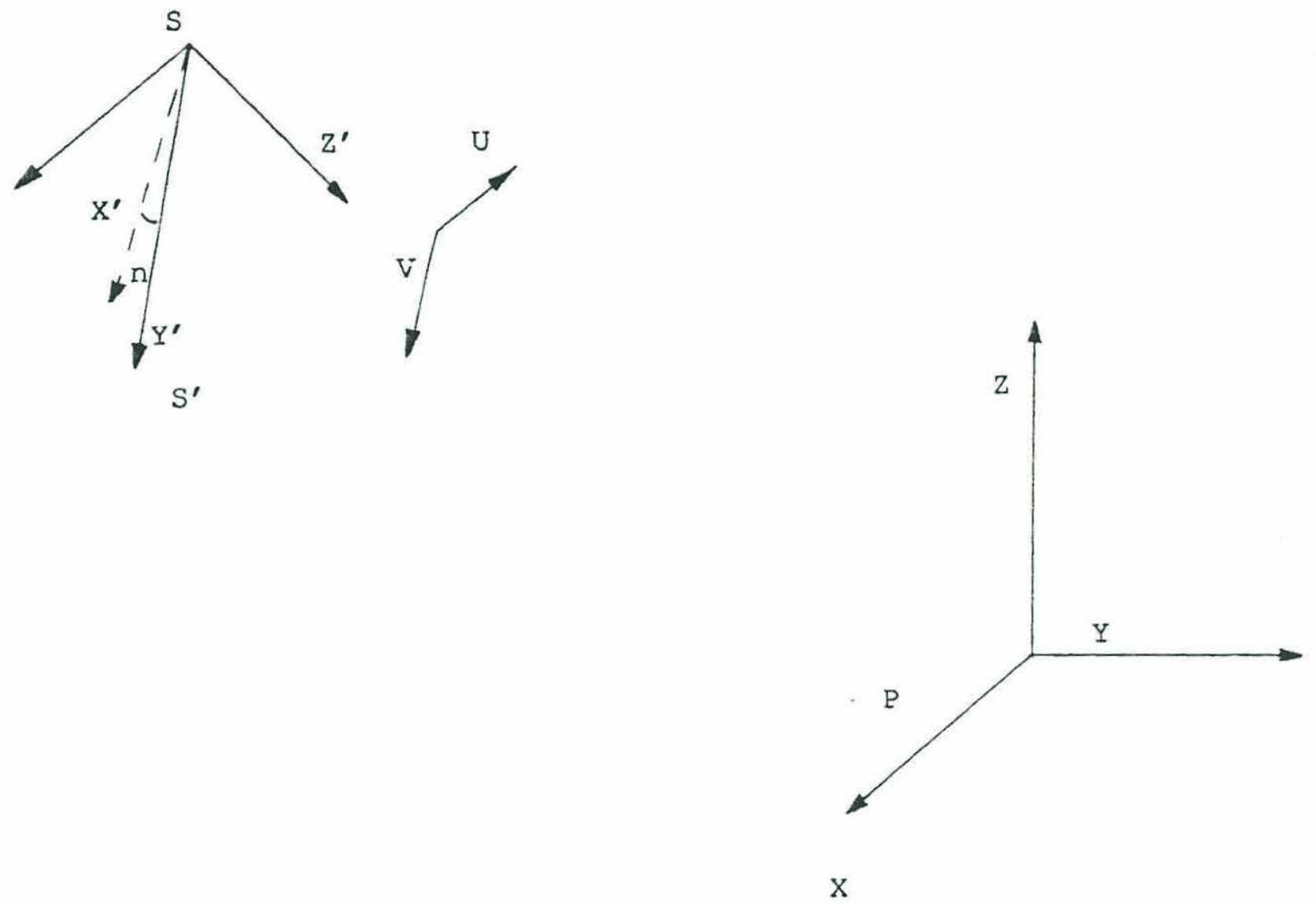

Swing Angle Rotation

Eigure 7

51 


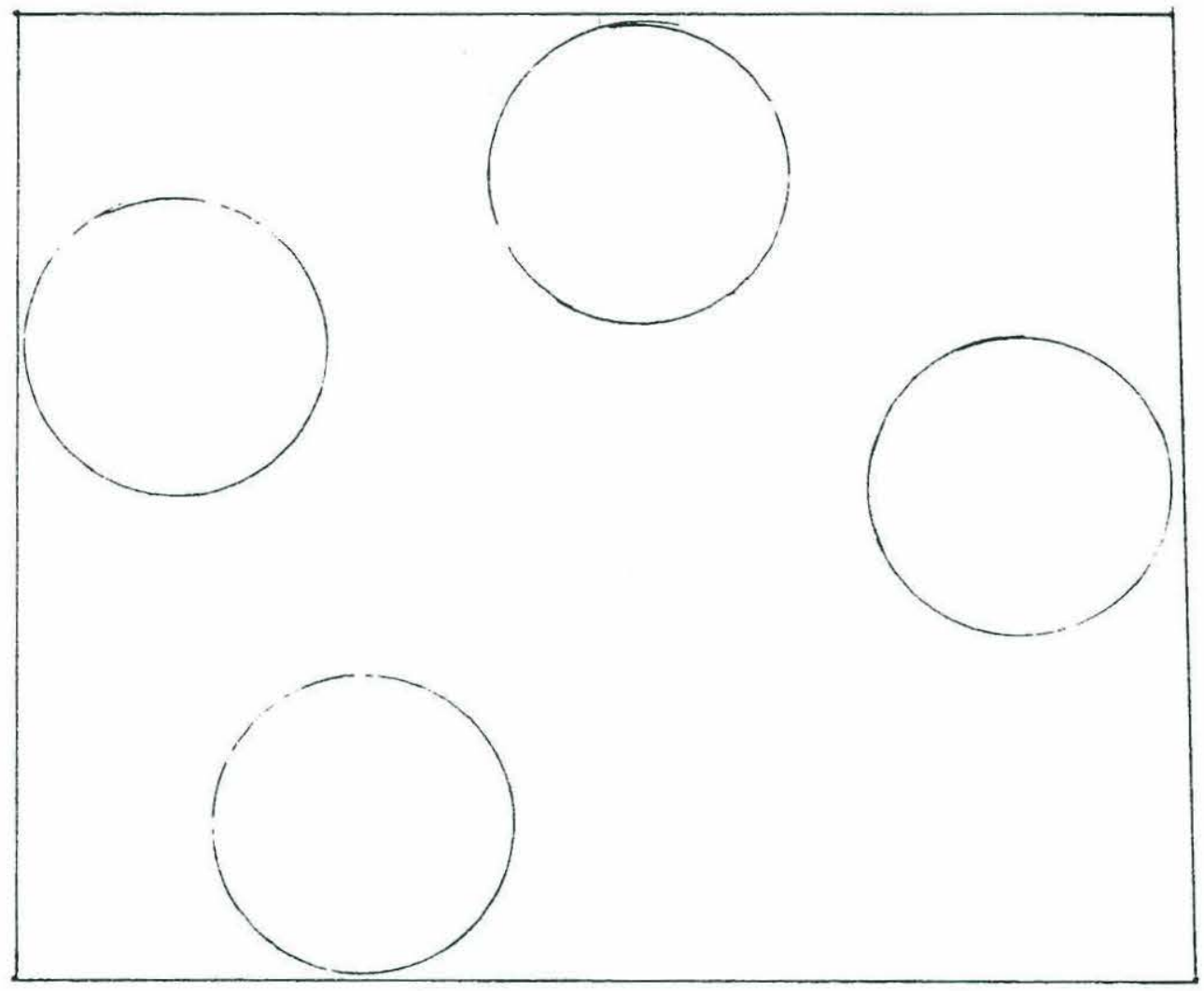

Possible Target Spot Location

Figure 8 


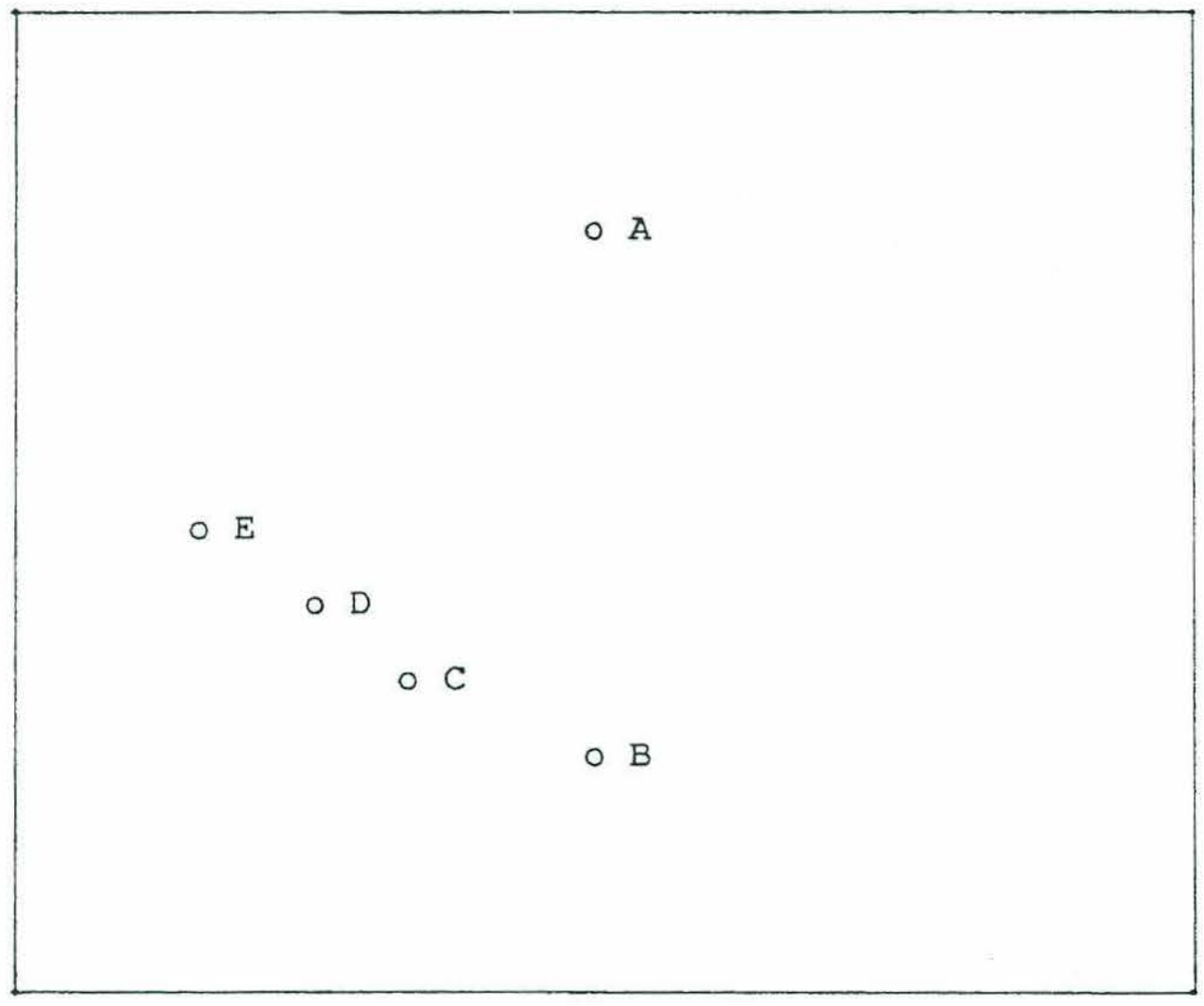

Test Target Base Plate

Eigure 9

53 


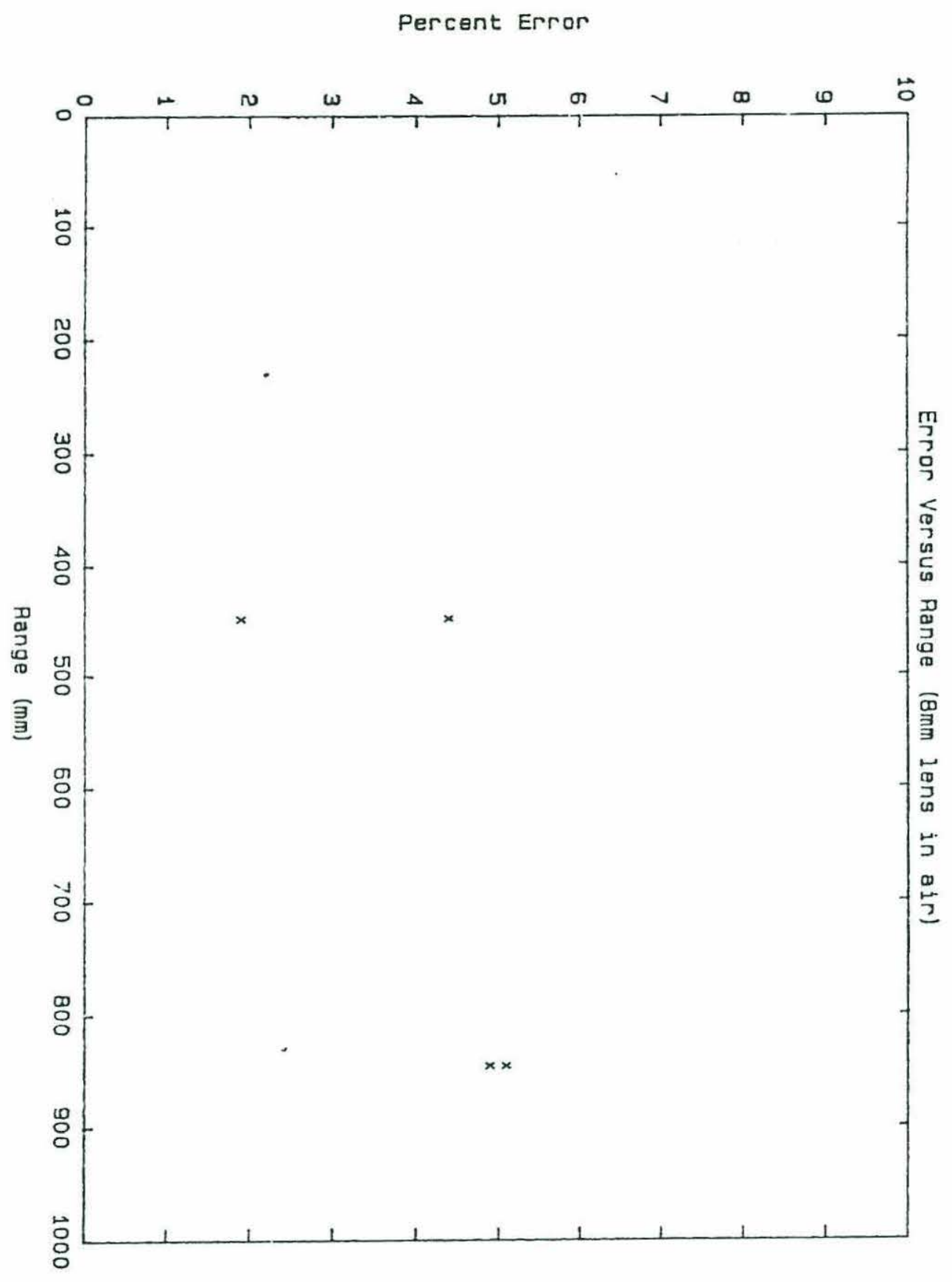

Eigure 10 


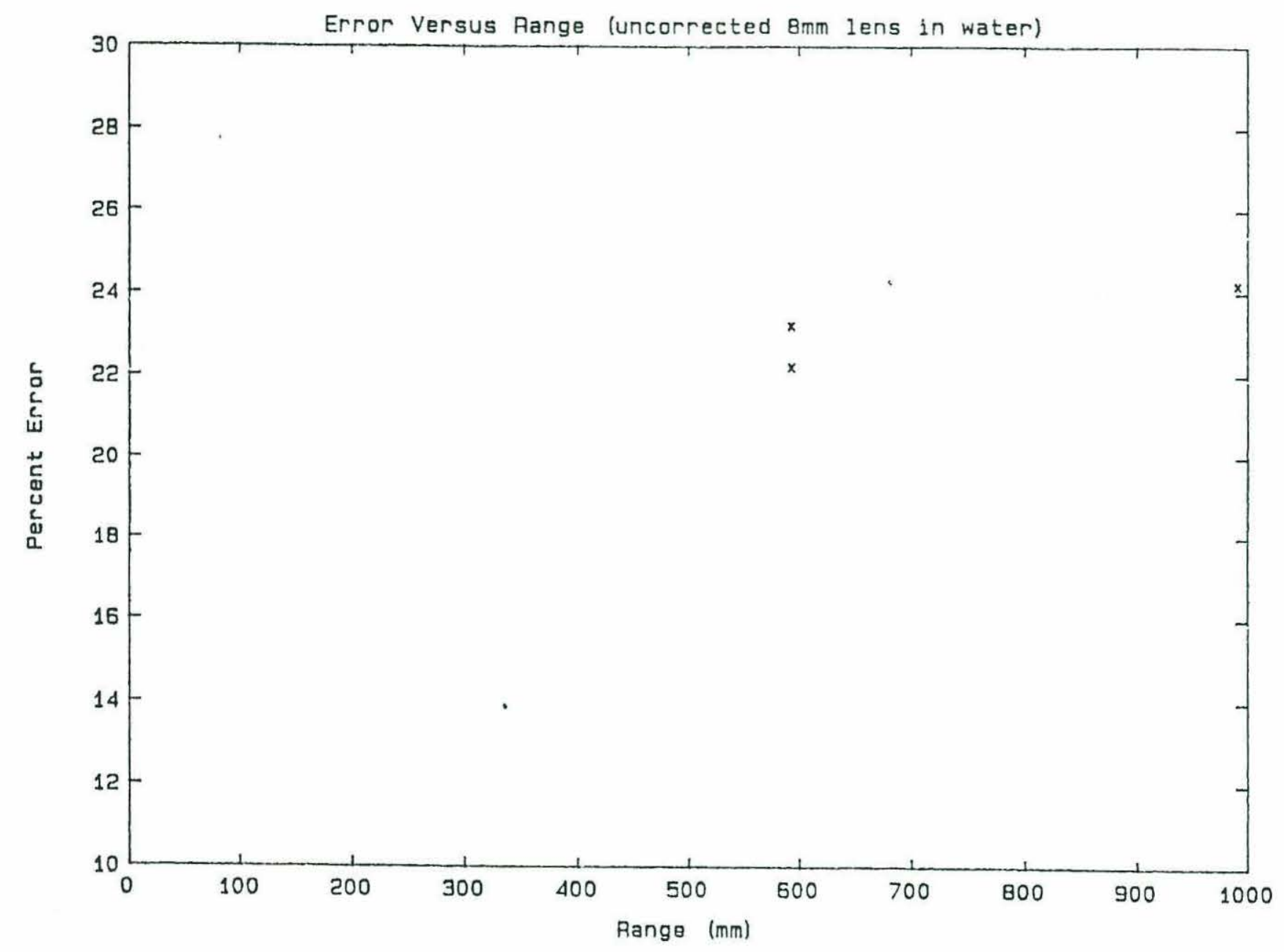




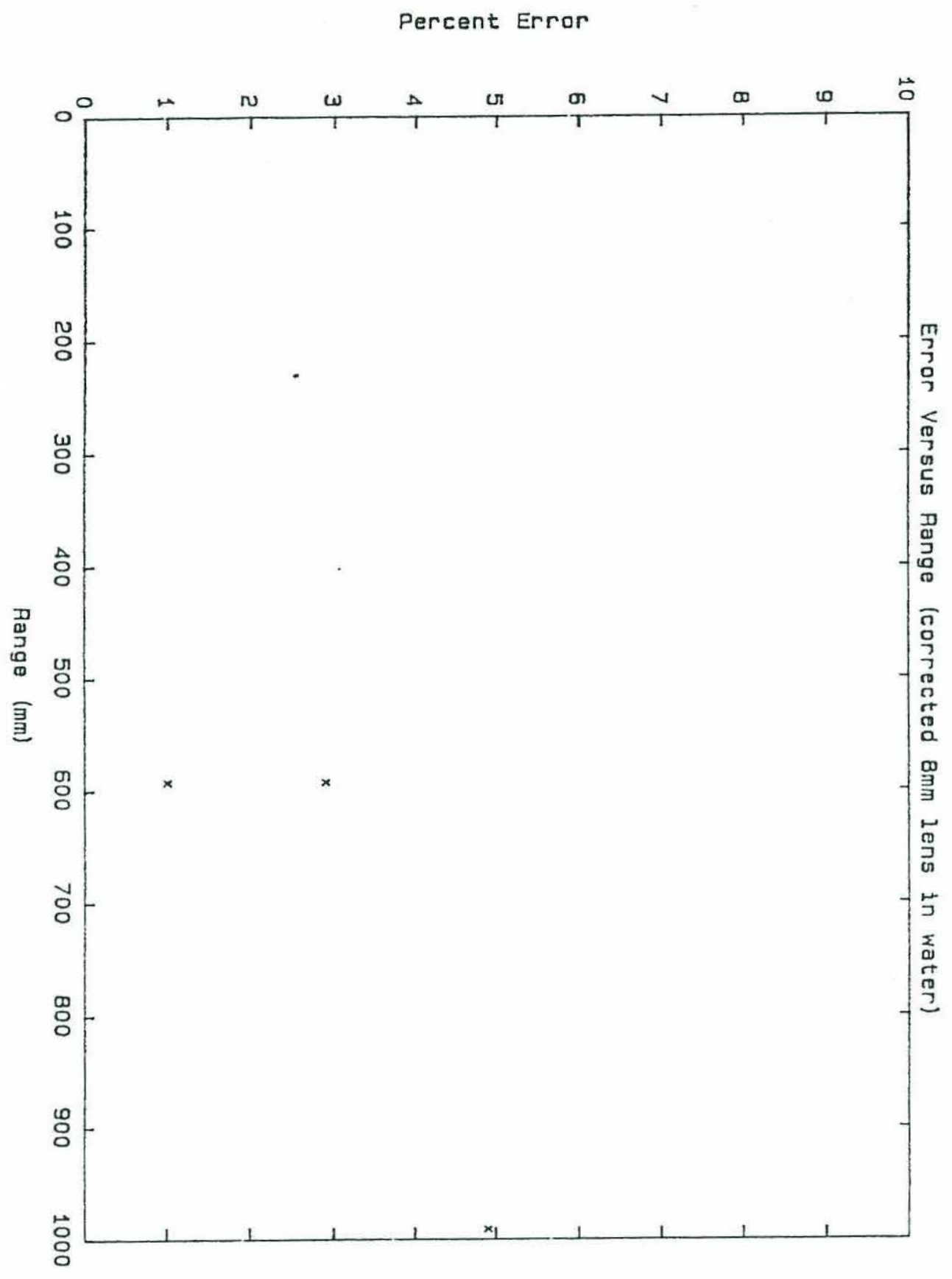

Eigure 12 


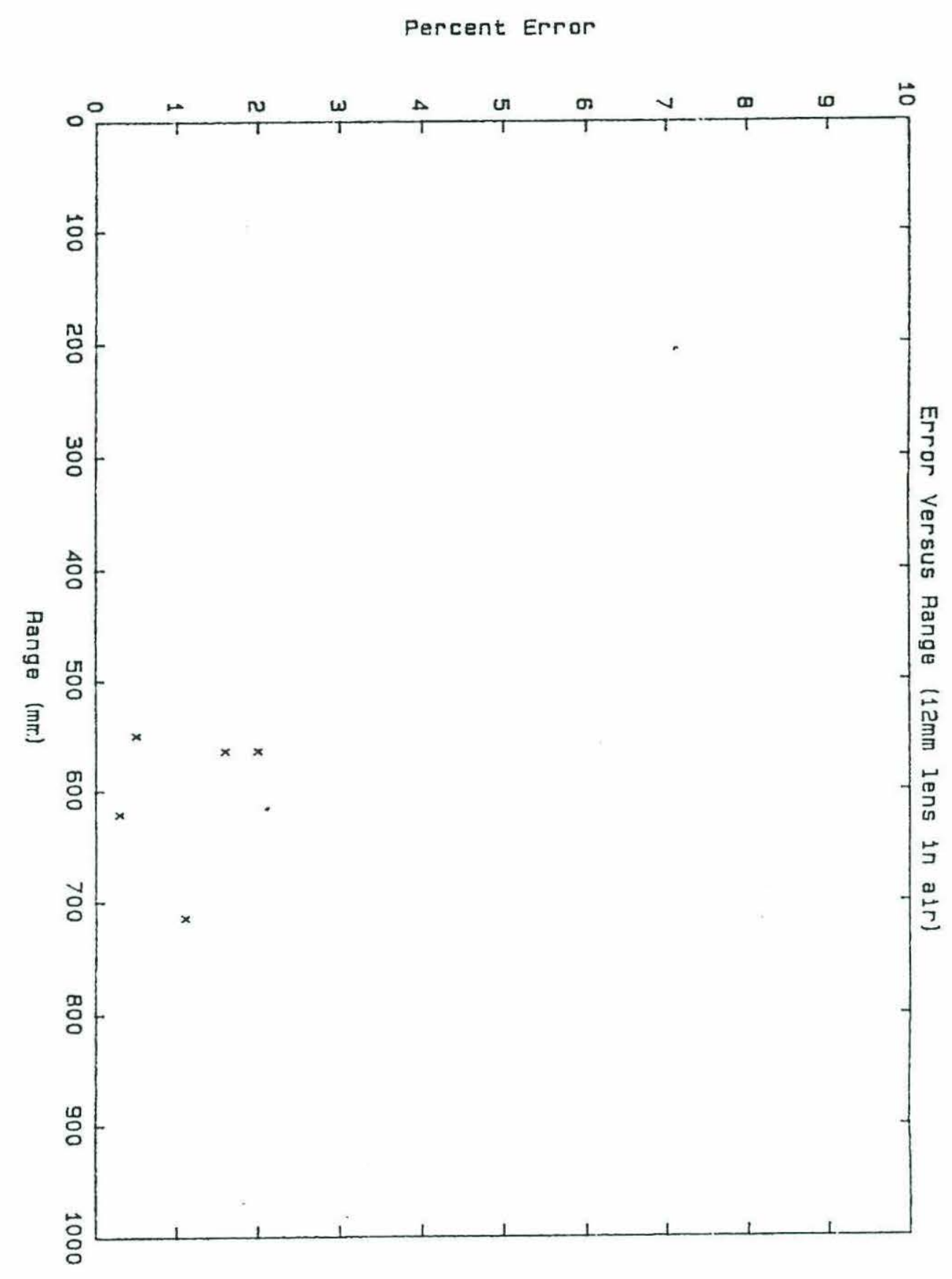

Figure 13 


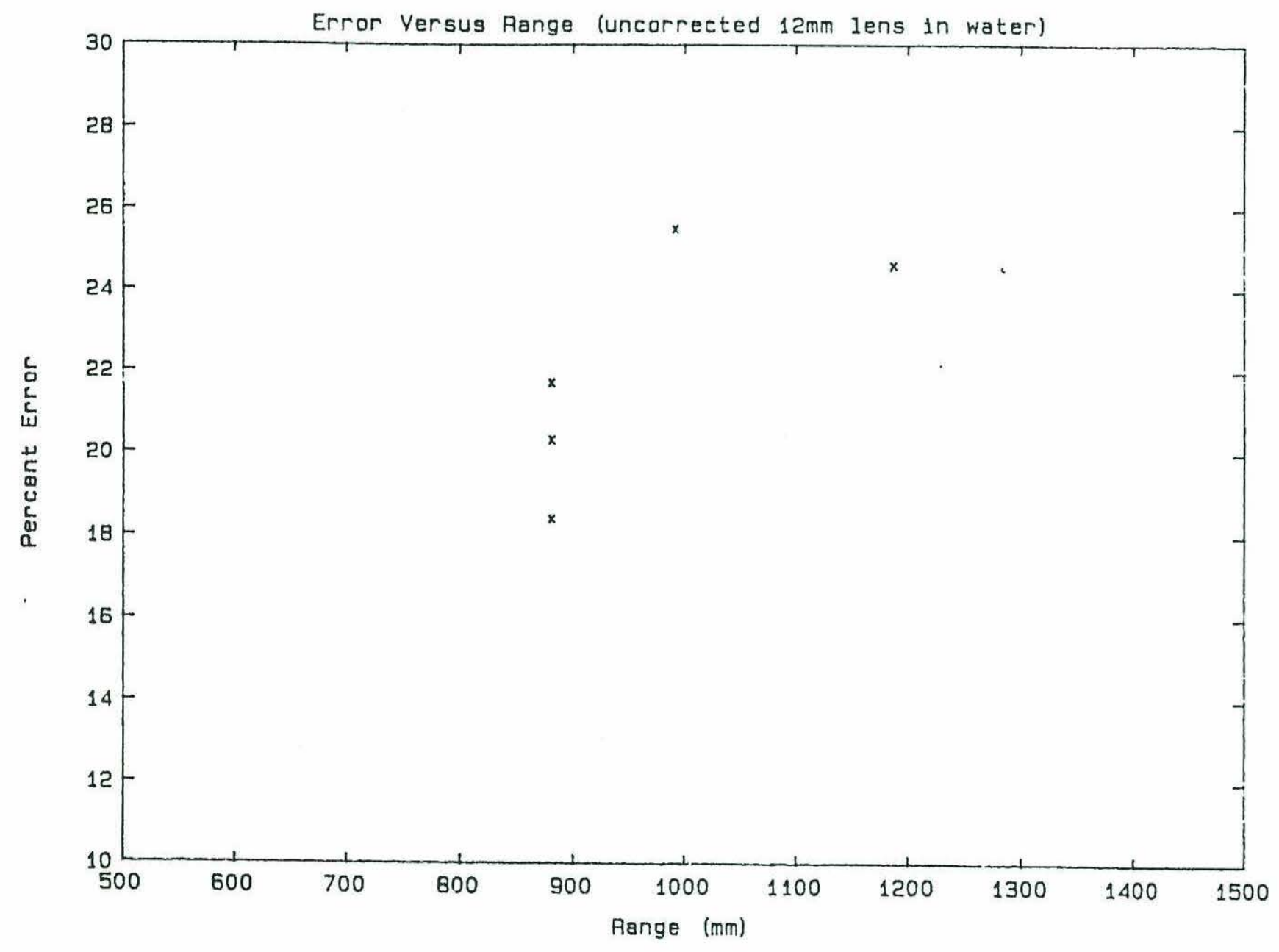




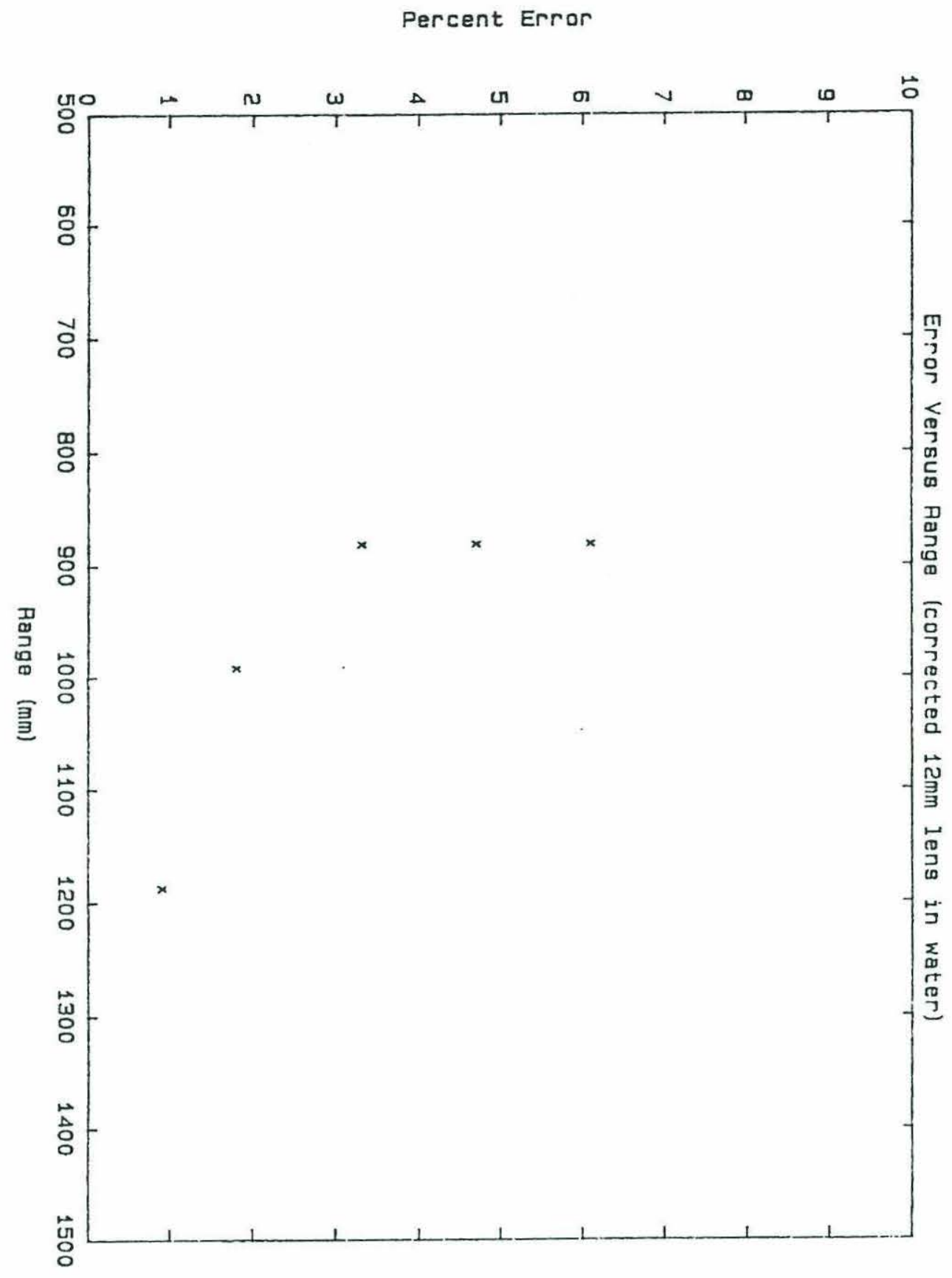

Eigure 15 
Appendix A $4.8 \mathrm{~mm}$ lens in air

$$
\begin{array}{cc}
\mathrm{k}_{1}= & 312.43537 \\
\mathrm{k}_{2}= & 184.29213 \\
\mathrm{x}_{\mathrm{i}-(\mathrm{mm})} & \\
-75.0 & \mathrm{y}_{\mathrm{i}}-(\mathrm{mm}) \\
0.0 & 150.0 \\
106.066 & 212.132 \\
75.0 & 75.0
\end{array}
$$

$\begin{array}{cc}\text { Test } 1: \text { camera at } & (0,0,-530 \mathrm{~mm}) \\ \underline{u}_{i \text { (vixel) }} & \mathrm{y}_{\mathrm{i} \text { (pixel) }} \\ 192 & 97 \\ 236 & 76 \\ 298 & 123 \\ 281 & 149\end{array}$

Result: $X_{s}=-55.9, Y_{s}=48.5, Z_{s}=-525.2$ 


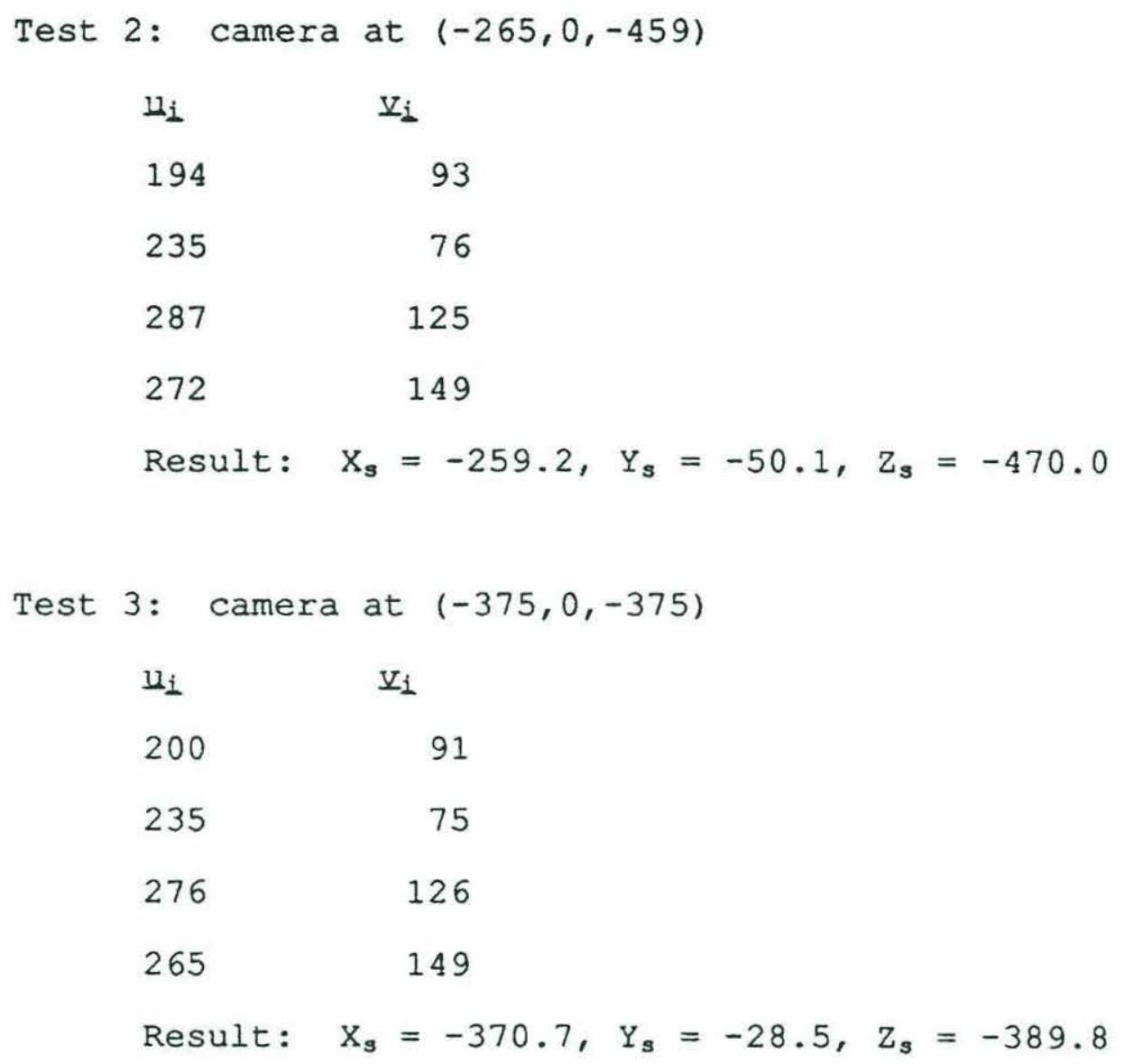


Test 5: camera at $(0,0,-1030)$

$\begin{array}{ll}\underline{u}_{i} & \underline{u}_{i} \\ 209 & 123 \\ 232 & 112 \\ 264 & 136 \\ 255 & 150\end{array}$

Result: $X_{s}=-22.9, Y_{s}=25.6, Z_{s}=-1051.6$

Test 6: camera at $(-515,0,-892)$

$\begin{array}{ll}u_{i} & \underline{y}_{i} \\ 211 & 122 \\ 231 & 112 \\ 259 & 137 \\ 252 & 150\end{array}$

Result: $\quad X_{s}=-516.5, Y_{s}=-41.0, Z_{s}=-874.8$

Test 7: camera at $(0,255,-530)$

는 $\quad \underline{y}_{i}$

$212 \quad 114$

$255 \quad 93$

$313 \quad 139$

$292 \quad 161$

Result: $X_{s}=4.5, Y_{s}=256.4, Z_{s}=-559.8$ 


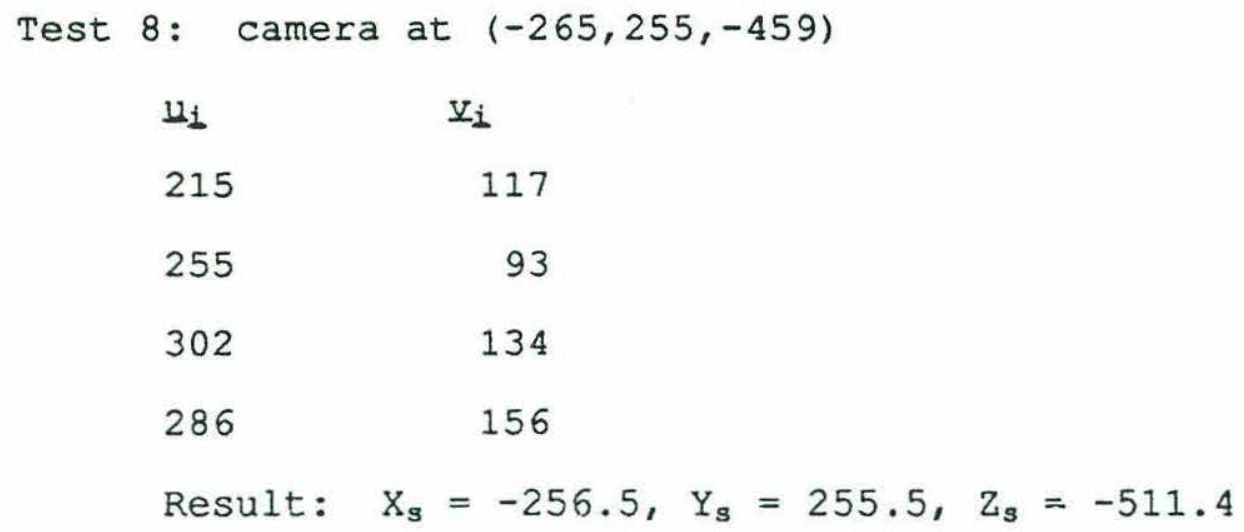




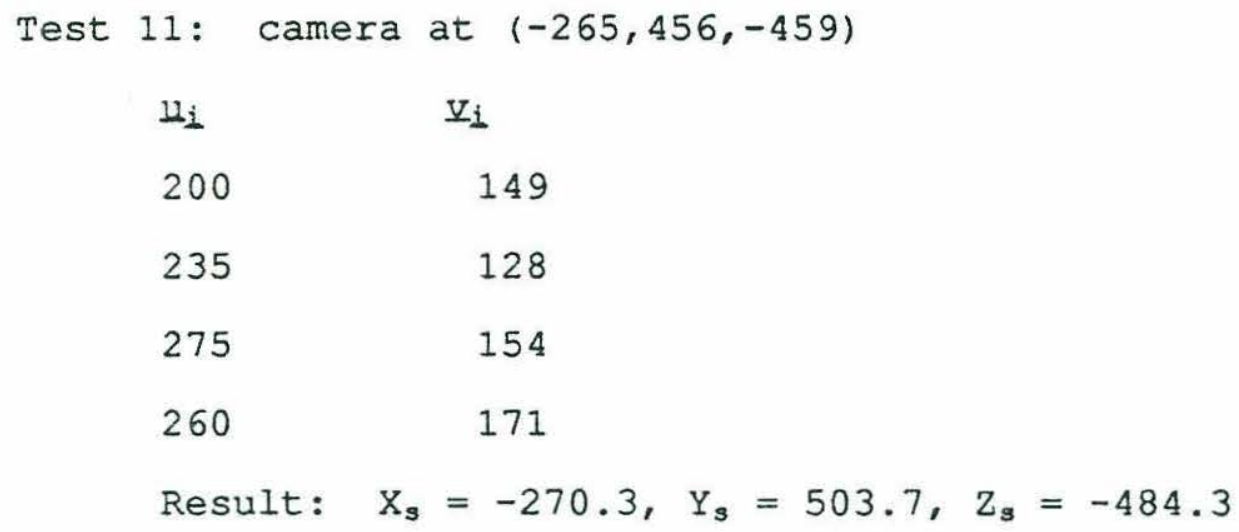




$$
\begin{aligned}
& \text { Appendix B 8mm lens in air } \\
& k_{1}=545.59157 \\
& k_{2}=323.03207
\end{aligned}
$$

Test 1: camera at $(-220,82,-381)$

\begin{tabular}{lr}
$\Perp_{i}$ & \multicolumn{1}{l}{} \\
165 & 94 \\
253 & 51 \\
362 & 152 \\
335 & 205
\end{tabular}

Result: $X_{s}=-214.2, Y_{s}=81.3, Z_{s}=-374.7$

Test 2: camera at $(-311,82,-311)$

$\mathrm{H}_{\mathrm{i}} \quad \mathrm{y}_{\mathrm{i}}$

$174 \quad 91$

$248 \quad 51$

$332 \quad 152$

$314 \quad 205$

Result: $X_{s}=-307.2, Y_{s}=83.6, Z_{s}=-291.6$ 


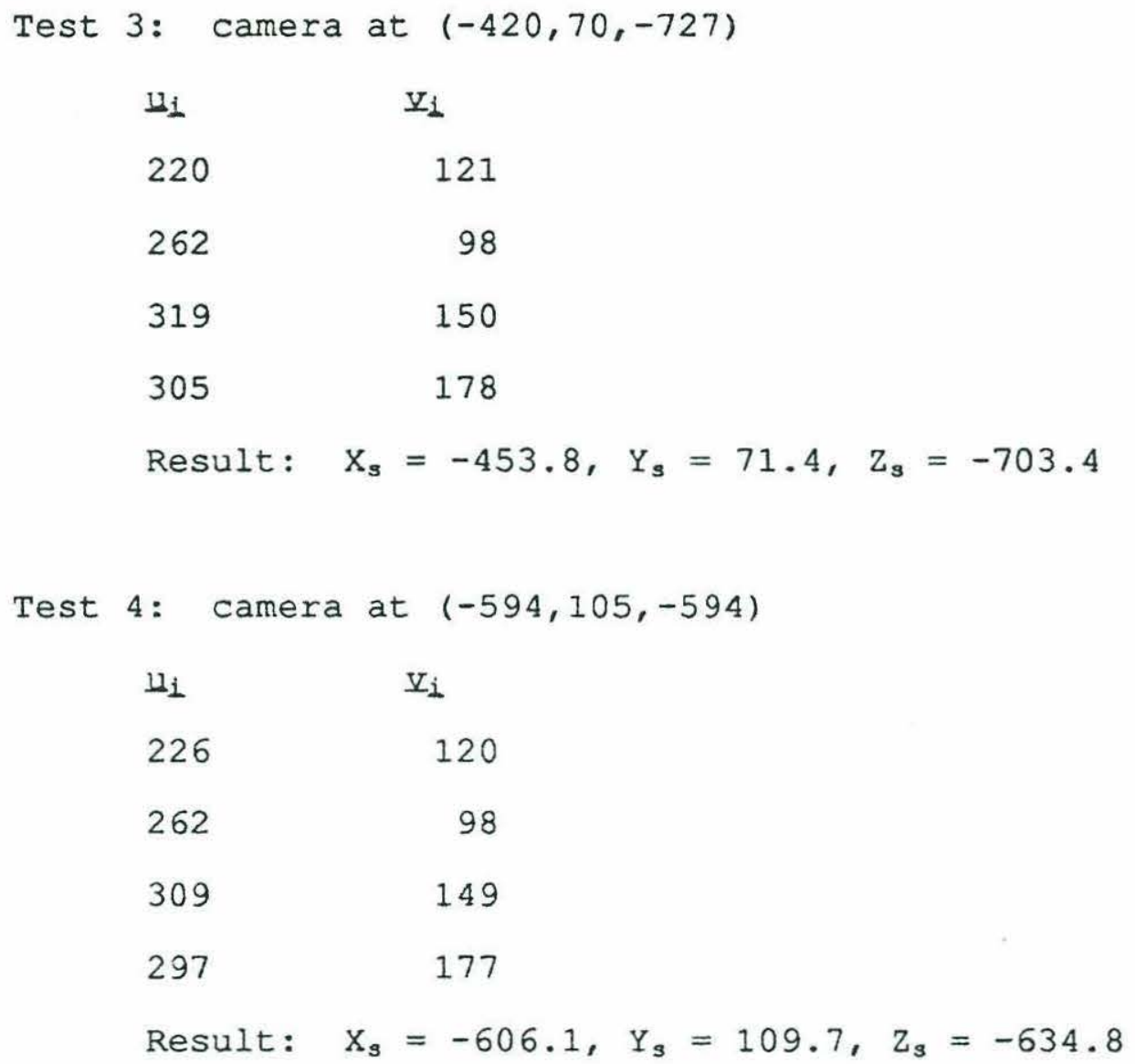

Result: $X_{s}=-606.1, Y_{s}=109.7, Z_{s}=-634.8$ 


\section{Appendix C $12 \mathrm{~mm}$ lens in air}

$$
\begin{aligned}
& k_{1}=818.38735 \\
& k_{2}=484.76785
\end{aligned}
$$

Test 1: camera at $(93,45,-540)$

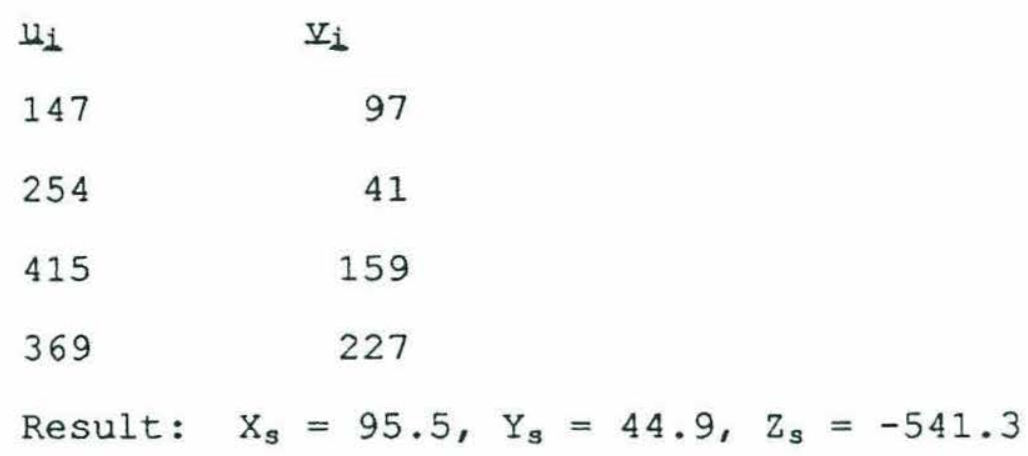




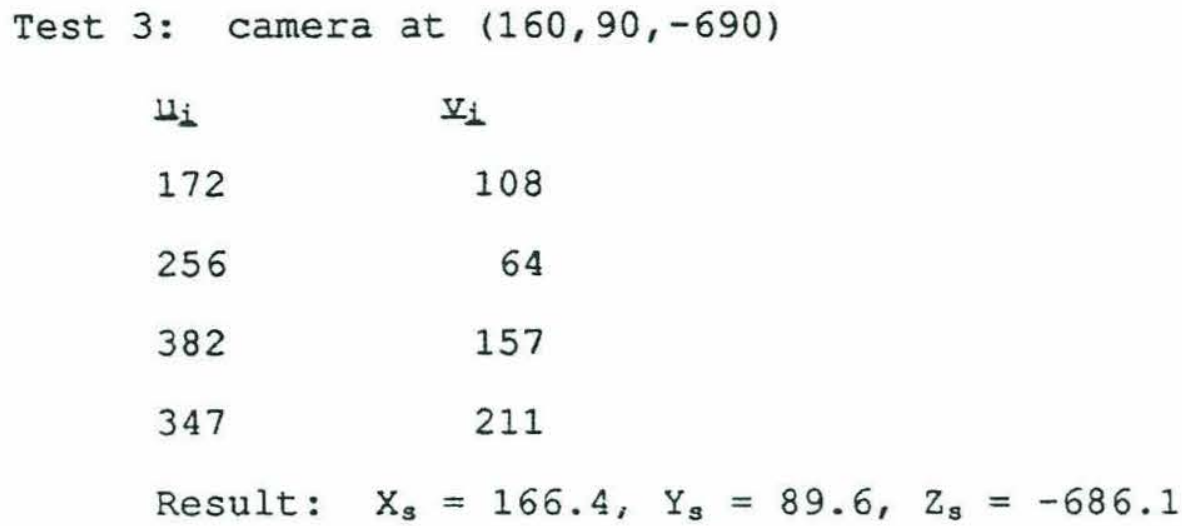




\section{Appendix D $8 \mathrm{~mm}$ lens in water}

Test 1: camera at $(-290,130,-502)$

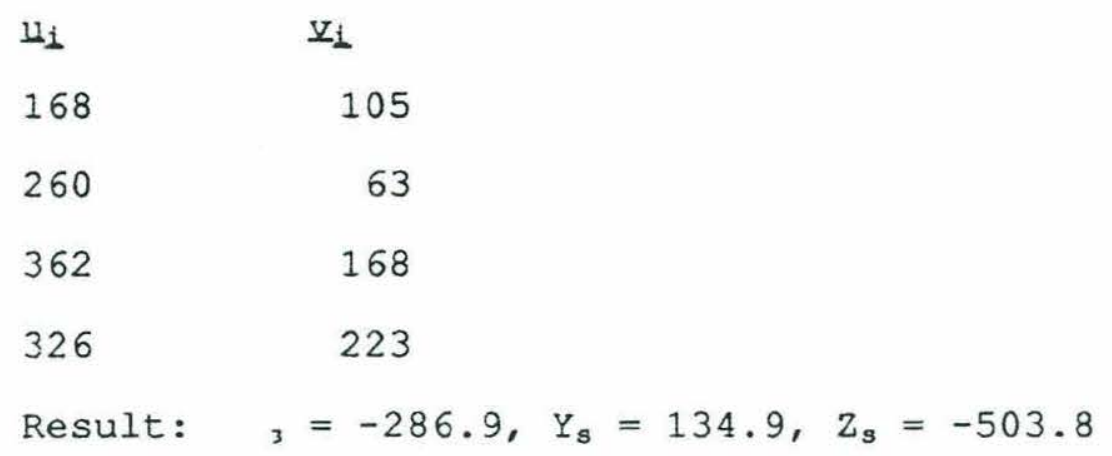

Result: $X_{s}=-409.6, Y_{s}=111.5, Z_{s}=-393.6$ 
Test 3: camera at $(200,105,-965)$

$\underline{u}_{i} \quad \underline{v}_{i}$

$215 \quad 123$

$270 \quad 97$

$342 \quad 160$

$315 \quad 192$

Result: $\quad X_{s}=244.3, Y_{s}=124.3, Z_{s}=-966.2$ 


\section{Appendix E $12 \mathrm{~mm}$ lens in water}

Test 1: came:a at $(200,105,-965)$

$\begin{array}{lc}\underline{H}_{i} & \mathrm{Y}_{i} \\ 163 & 87 \\ 248 & 48 \\ 358 & 143 \\ 318 & 192 \\ \text { Result: } & \mathrm{X}_{\mathbf{s}}=187.6, \mathrm{Y}_{\mathbf{s}}=95.9, \mathrm{Z}_{s}=-955.2\end{array}$

Test 2: camera at $(200,105,-1165)$

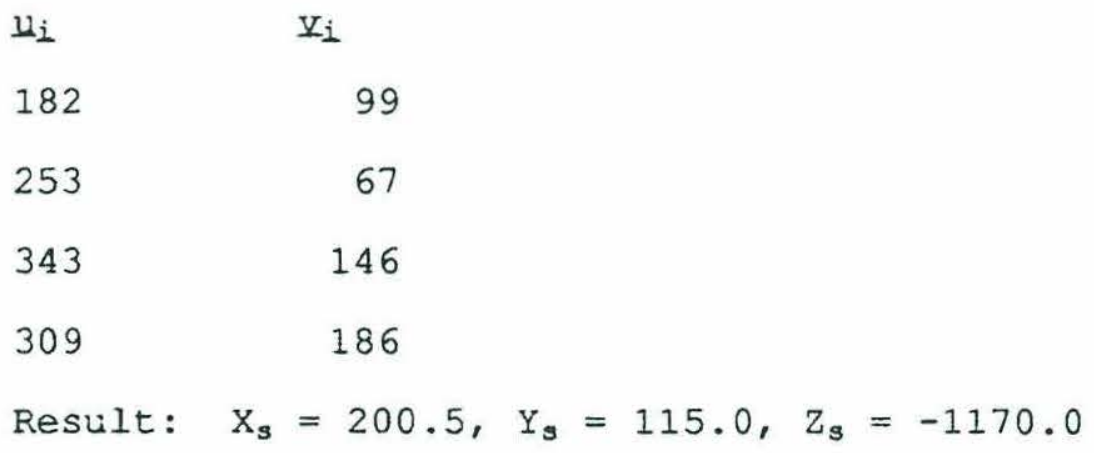


Test 3 : camera at $(-440,30,-762)$

$\begin{array}{lc}\Perp_{i} & \underline{y}_{1} \\ 163 & 76 \\ 250 & 36 \\ 351 & 142 \\ 315 & 194 \\ \text { Result: } & \mathrm{X}_{s}=-473.1, Y_{s}=5.1, Z_{s}=-764.3\end{array}$

Test $4:$ camera at $(-622,30,-622)$

$u_{i} \quad \underline{x}_{i}$

$187 \quad 75$

$260 \quad 36$

$338 \quad 142$

$309 \quad 194$

Result: $X_{s}=-643.6, Y_{s}=43.8, Z_{s}=-607.6$

Test 5: camera at $(-762,30,-440)$

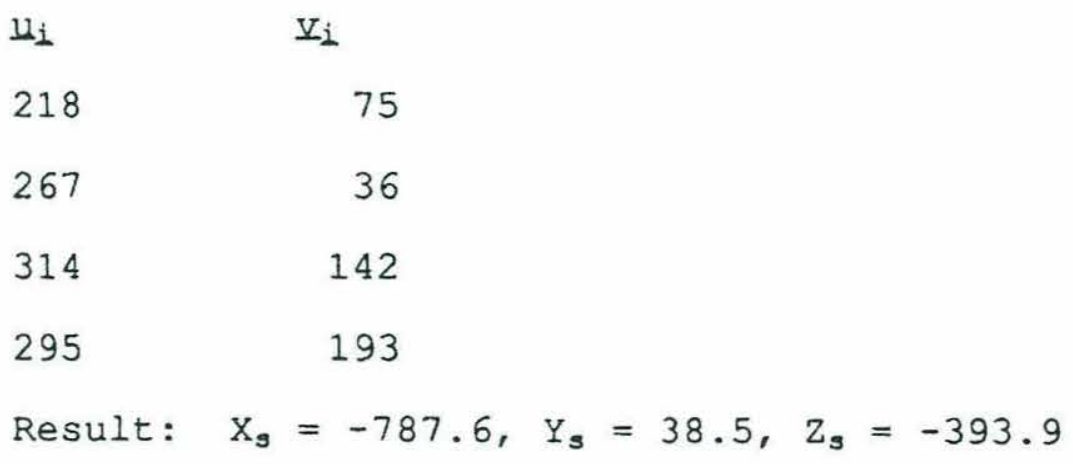




\section{Bibliography}

Duda, R. O., P. E. Hart, Pattern Recognition and Scene Analysis, New York, Wiley, 1973.

Fischler, M. A., R. C. Bolles, "Random Sample Consensus: A Paradigm for Model Fitting with Applications to Image Analysis and Automated Cartography", Proceedings Image Understanding Workshop, April 1980, pp 71-88.

Ganapathy, S., "Decomposition of Transformation Matrices for Robot Vision", Proceedings of the First International Conference on Robotics, March 1984, pp 130-139.

George, J. D., G. I. Lythgoe, J. N. Lythgoe, Underwater Photography and Television for Scientists, Oxford, Clarendon Press, 1985.

Haralick, R. M., "Using Perspective Transformations in Scene Analysis", Computer Graphics and Image Processing, $13(1980)$, pp 191-221.

Horaud, R., B. Conio, O. Leboulleux, B. Lacolle, "An Analytic Solution for the Perspective 4-Point Problem", Computer Vision, Graphics, and Image Processing, 47 (1989), No 1, pp 33-44.

Huang, T. S., R. Y. Tsai, "Image Sequence Analysis: Motion Estimation", Image Sequence Analysis, New York, SpringerVerlag, 1981, pp 1-18.

Kanattani, K., "Camera Rotation Invariance", Computer Vision, Graphics, and Image Processing, 39(1987), No 3, pp 328354 .

Lowe, D. G., "Solving for the Parameters of Object Models From Image Descriptions", Proceedings Image Understanding Workshop, April 1980, pp 121-127.

McNeil, G. T., "Underwater Photography", Proceedings of the Society of Photo-optical Instrumentation Engineers, 24 (1971), pp 117-131.

Nasr, H., B. Bhanu, "Landmark Recognition for Autonomous Mobile Robots", Proceedings IEEE International Conference on Robotics and Automation, 1988, pp 1218-1223. 
Roach, J. E., J. K. Aggarwal, "Determining the Movement of Objects from a Sequence of Images", IEEE Transact ions on PAMI, November 1976, pp 554-562.

Sugihara, K., "Some Location Problems for Robot Navigation Using a Single Camera", Computer Vision, Graphics, and Image Processing, 42(1988), No 1, pp 112-129.

Sutherland, I. E., "Three-Dimensional Data Input by Tablet", Proceedings IEEE, 621(1974), No 4, pp 453-461. 